\title{
Exponentially small splitting of separatrices for perturbed integrable standard-like maps
}

\author{
Amadeu Delshams and Rafael Ramírez-Ros \\ Departament de Matemàtica Aplicada I \\ Universitat Politècnica de Catalunya \\ Diagonal 647, 08028 Barcelona, Spain
}

Revised version, August 18, 1997*

\section{Summary}

The splitting of separatrices for the standard-like maps

$$
F(x, y)=\left(y,-x+\frac{2 \mu y}{1+y^{2}}+\varepsilon V^{\prime}(y)\right), \quad \mu=\cosh h, \quad h>0, \quad \varepsilon \in \mathbb{R}
$$

is measured. For even entire perturbative potentials $V(y)=\sum_{n>2} V_{n} y^{2 n}$ such that $\widehat{V}(2 \pi) \neq 0$, where $\widehat{V}(\xi)=\sum_{n>2} V_{n} \xi^{2 n-1} /(2 n-1)$ ! is the Borel transform of $V(y)$, the following asymptotic formula for the area $A$ of the lobes between the perturbed separatrices is established

$$
A=8 \pi \widehat{V}(2 \pi) \varepsilon \mathrm{e}^{-\pi^{2} / h}\left[1+\mathrm{O}\left(h^{2}\right)\right] \quad\left(\varepsilon=\mathrm{o}\left(h^{6} \ln ^{-1} h\right), h \rightarrow 0^{+}\right) .
$$

This formula agrees with the one provided by the Melnikov theory, which cannot be applied directly, due to the exponentially small size of $A$ with respect to $h$.

AMS Subject Classification (1991): Primary 34C37; Secondary 33E05, 34C11, 34C20

\section{Introduction and main results}

\section{The problem}

As already noticed by Poincaré more than a century ago [Poi99], while he was studying perturbations of integrable Hamiltonian systems - the "fundamental problem of the mechanics" - , the separatrices associated to integrable systems near simple resonances

\footnotetext{
*To appear in J. Nonlinear Sci.
} 
split under general perturbations. Moreover, the amount of stochastic behaviour near these resonances is measured by the size of such splitting of separatrices, which turns out to be exponentially small with respect to the parameter of perturbation. Thus, this phenomenon could not be detected by the classical perturbation theory, and it remained unsolved for a long time.

It was not until the last decade that the effective measure of such splitting was finally proved by several authors [HMS88, GLT91, DS92, Ang93, Gel93, Tre97, Sau95], but for some concrete problems. For more general systems, exponentially small upper estimates for the splitting of separatrices in Hamiltonian systems were given in several papers [Nei84, Fon93, Sim94, Fon95, FS96], and very recently, asymptotic expressions or lower estimates [Gal94, Gel97, DGJS97, DS97, RW97] have been obtained by several means.

Hamiltonian systems with two degrees of freedom near resonances can also be studied via the induced Poincaré maps, giving rise to different models of families of analytic area preserving maps close to the identity. For such families of planar maps, exponentially small upper estimates of the splitting of separatrices have been obtained by several authors [Nei84, FS90, Fon95, Gel96], whereas the effective measure of the splitting size has only been formulated for some celebrated entire standard-like maps by V. Lazutkin and co-workers [Laz84, LST89, GLT91, GLS94], as well as by D. Treschev [Tre96a].

However, a complete proof of the asymptotic formulae for the splitting of separatrices for these entire maps has not been published yet, in spite of the intensive efforts devoted to it. An important complexity arises from the fact that these celebrated maps (like the standard map or the Hénon map), cannot be easily expressed as close enough (also in the complex field) to an integrable map defined on the whole plane.

In this paper we start with an integrable standard-like map, which possesses separatrices associated to a fixed point with characteristic multipliers $\mathrm{e}^{ \pm h}$. The parameter $h>0$ will be assumed small, and therefore the fixed point is weakly hyperbolic. Next, a general entire standard-like perturbation is considered. Since we want to deal with analytic perturbations also with respect to $h$, the size $\varepsilon$ of the perturbation is assumed to be of the form $\varepsilon=h^{p}$ for some $p \geq 0$. Then, for $p>6$, an asymptotic formula is obtained for the splitting of separatrices, which is exponentially small in $h$. (Lower values of $p$ are also discussed numerically.)

To the best of our knowledge, this is the first time that such a formula is rigorously proved for a discrete dynamical system. The proof is based on a rigorous justification of the Melnikov method for maps. This methodology follows an approach suggested by V. Lazutkin [Laz84], which has been already developed for rapidly forced flows close to integrable ones [DS92, DS97].

\section{The model}

In the present paper we consider the family $F: \mathbb{R}^{2} \rightarrow \mathbb{R}^{2}$ of standard-like maps

$$
F(x, y)=\left(y,-x+\frac{2 \mu y}{1+y^{2}}+\varepsilon V^{\prime}(y)\right), \quad \mu=\cosh h, \quad h>0, \quad \varepsilon \in \mathbb{R}
$$




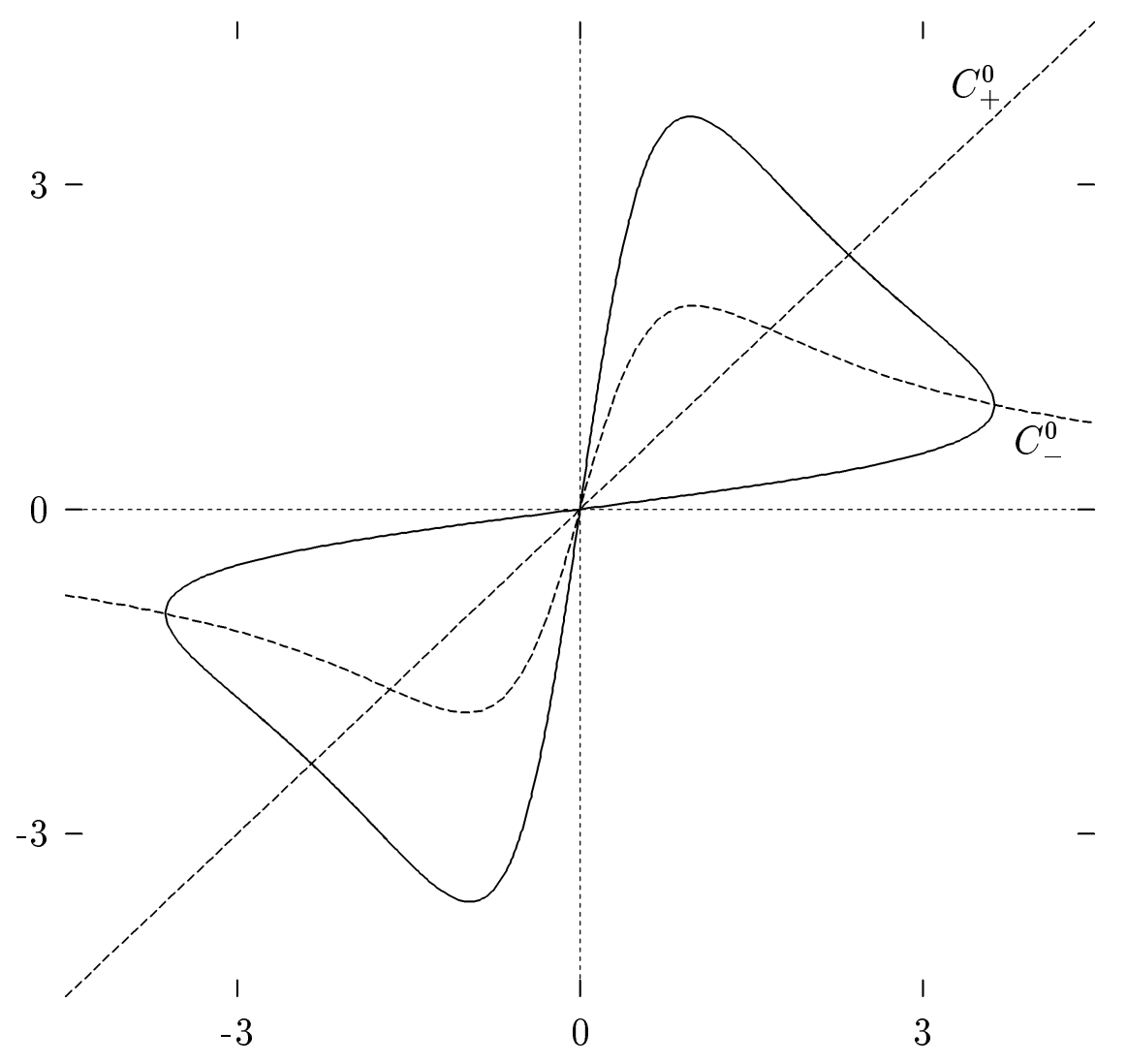

Figure 1: The zero level of $H^{0}$ for $h=2$.

where the function $V: \mathbb{C} \rightarrow \mathbb{C}$ (which will be called the perturbative potential) is determined by imposing $V(0)=0$. We shall assume that $V(y)$ is an even entire function such that $V^{\prime \prime}(0)=0$. Its Taylor expansion will be denoted by

$$
V(y)=\sum_{n \geq 2} V_{n} y^{2 n}
$$

Introducing

$$
\mathcal{L}=\mathcal{L}^{0}+\varepsilon \mathcal{L}^{1}, \quad \mathcal{L}^{0}(x, X)=-x X+\mu \log \left(1+X^{2}\right), \quad \mathcal{L}^{1}(x, X)=V(X),
$$

it is immediate to check that $\mathcal{L}(x, X)$ is a twist generating function for the map (1.1), i.e., $F(x, y)=(X, Y)$ if and only if $y=-\partial_{1} \mathcal{L}(x, X)$ and $Y=\partial_{2} \mathcal{L}(x, X)$. As a consequence, every standard-like map of the family (1.1) is a twist map, and in particular, is an area preserving map.

Twist maps are frequently used as good models for return maps associated to Hamiltonian systems with two degrees of freedom, as well as for motion inside particle accelerators. A discussion of the adequacy of our model (1.1) for representing a physical system can be found in [McM71, Las78]. 
The unperturbed map is the so-called McMillan map [McM71]

$$
F^{0}(x, y)=\left(y,-x+\frac{2 \mu y}{1+y^{2}}\right),
$$

which is integrable, with a polynomial first integral given by

$$
H^{0}(x, y)=\left(x^{2}-2 \mu x y+y^{2}+x^{2} y^{2}\right) / 2 \gamma, \quad \gamma=\sinh h .
$$

The origin is a hyperbolic fixed point where $\operatorname{Spec}\left[\mathrm{d} F^{0}(0,0)\right]=\left\{\lambda, \lambda^{-1}\right\}$ with $\lambda=\mathrm{e}^{h}$, that is, $h$ is the characteristic exponent of the origin. Therefore, for small $h>0$, the origin is weakly hyperbolic. Moreover, the McMillan map has a separatrix to the origin in the first quadrant contained in the zero level of the first integral $H^{0}$, see figure 1 , which can be parameterized by

$$
z^{0}(t)=\left(x^{0}(t), y^{0}(t)\right)=\left(\xi^{0}(t-h / 2), \xi^{0}(t+h / 2)\right), \quad \xi^{0}(t)=\gamma \operatorname{sech} t .
$$

This parameterization will be called natural since $F^{0}\left(z^{0}(t)\right)=z^{0}(t+h)$, a fact that can be checked simply by noting that $\xi^{0}(t)$ is a homoclinic solution of the difference equation

$$
\xi^{0}(t+h)+\xi^{0}(t-h)=f^{0}\left(\xi^{0}(t)\right), \quad f^{0}(y)=\frac{2 \mu y}{1+y^{2}},
$$

and that $F^{0}(x, y)=\left(y,-x+f^{0}(y)\right)$. All these properties of the McMillan map can be found in [GPB89, DR96].

From now on, given the characteristic exponent $h>0$, the notations

$$
\mu=\cosh h, \quad \gamma=\sinh h, \quad \lambda=\mathrm{e}^{h}
$$

will be used without further comment. Besides, to avoid a cumbersome notation, the dependence of all functions on $h$ and $\varepsilon$ is not explicitly written. To end the conventions about notation, the superscript " 0 " will denote an unperturbed quantity, that is, $\varepsilon=0$.

When the perturbation is taken into consideration, the qualitative behaviour of the map (1.1) changes drastically. For fixed $h>0$, and $\varepsilon \neq 0$ small enough, the perturbed invariant curves cross and the map $F$ becomes non-integrable for any non-constant entire perturbative potential $V(y)$ [DR96].

The standard-like map (1.1) is reversible with respect to the involutions

$$
R_{+}(x, y)=(y, x), \quad R_{-}(x, y)=\left(x,-y+\frac{2 \mu x}{1+x^{2}}+\varepsilon V^{\prime}(x)\right)=\left(F R_{+}\right)(x, y)
$$

i.e., $F^{-1}=R_{ \pm} F R_{ \pm}$. Since $R_{ \pm}\left(\mathcal{W}^{\mathrm{u}}\right)=\mathcal{W}^{\mathrm{s}}$, in order to find a homoclinic orbit, it is sufficient to search for points $z_{ \pm}$of the unstable curve that intersect the curves $C_{ \pm}:=$ $\left\{z \in \mathbb{R}^{2}: R_{ \pm}(z)=z\right\}$, see figure 2 . Such an orbit is called a reversible homoclinic orbit. For $\varepsilon=0$, the unstable curve intersects (transversely) the curves $C_{ \pm}^{0}$ just in four points, excluding the origin, see figure 1 . Thus, for small enough $\varepsilon$ there exist at least four 


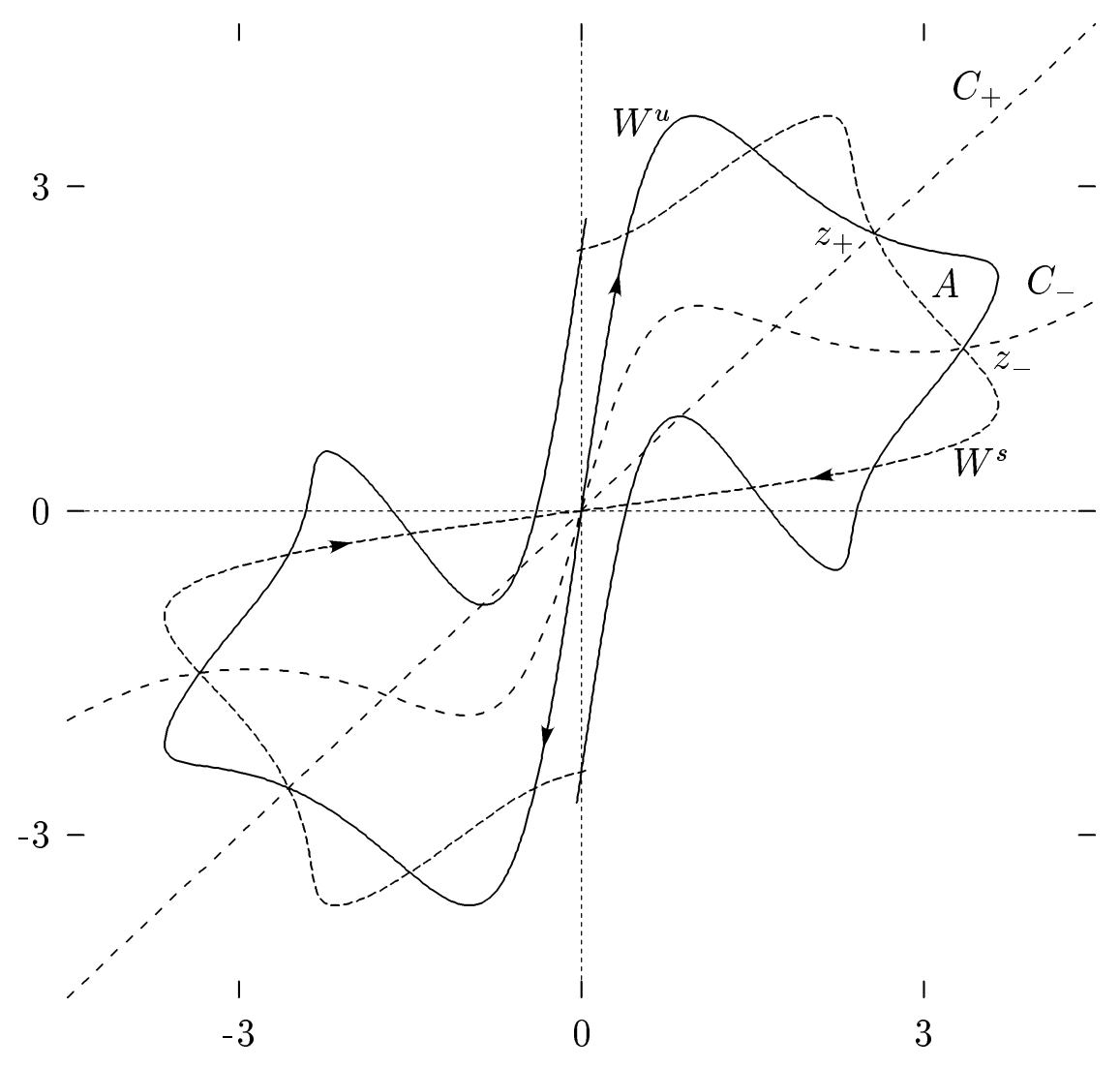

Figure 2: The homoclinic points $z_{ \pm}$and the lobe area $A$ for $V^{\prime}(y)=y^{3}, h=2$ and $\varepsilon=0.025$.

primary reversible homoclinic orbits, two on the first quadrant, and their symmetrical pairs in the third one. For general perturbations, these four symmetric orbits will be the only primary homoclinic orbits, and in this case, our measure of the splitting size will be the algebraic ${ }^{1}$ area $A$ of the lobe that remains between the two invariant curves from $z_{+}$to $z_{-}$, see figure 2 again. It should be noted that this lobe area $A$ is a symplectic invariant, that is, it does not depend on the symplectic coordinates used, and all the lobes have the same (geometric) area: $|A|$.

\footnotetext{
${ }^{1}$ The term algebraic means that $A$ can take negative values. The usual geometric area is $|A|$. The sign of $A$ can be used to know the way in which the perturbed curves cross: if $\eta=\eta^{\mathrm{u}}-\eta^{\mathrm{s}}$, where $\eta^{\mathrm{u}, \mathrm{s}}$ is the path from $z_{+}$to $z_{-}$inside $\mathcal{W}^{\mathrm{u}, \mathrm{s}}$, then $A>0$ if and only if $\eta$ is traveled clockwise, like in figure 2 .
} 


\section{The results}

A direct application of the Melnikov theory [DR96, DR97a] gives the following formula for the lobe area

$$
A=\varepsilon \int_{0}^{h / 2} M(t) \mathrm{d} t+\mathrm{O}\left(\varepsilon^{2}\right)
$$

where $M(t)=M(t, h)$ is the celebrated Melnikov function:

$$
M(t)=\sum_{n \in \mathbb{Z}} \mathrm{d} H^{0}\left(F^{0}\left(z^{0}\left(t_{n}\right)\right)\right) F^{1}\left(z^{0}\left(t_{n}\right)\right) \quad t_{n}=t+h n,
$$

$F^{1}$ being the perturbation, i.e., $F=F^{0}+\varepsilon F^{1}$. Since $F$ is a twist map generated by the generating function $\mathcal{L}=\mathcal{L}^{0}+\varepsilon \mathcal{L}^{1}$ given in (1.3), there exists a function $L(t)=L(t, h)$ (called the Melnikov potential [DR96]) such that $M(t)=L^{\prime}(t)$, given by

$$
L(t)=\sum_{n \in \mathbb{Z}} \mathcal{L}^{1}\left(x^{0}\left(t_{n}\right), x^{0}\left(t_{n+1}\right)\right), \quad t_{n}=t+h n .
$$

Hence, using equations (1.3) and (1.6), we can express the Melnikov function and the Melnikov potential as

$$
M(t)=L^{\prime}(t), \quad L(t)=\sum_{n \in \mathbb{Z}} f(t+h n), \quad f(t)=f(t, h)=V(\gamma \operatorname{sech}(t+h / 2)) .
$$

This formula shows clearly that $L(t), M(t)$ are doubly periodic functions with periods $h$ and $2 \pi \mathrm{i}$ ( $\pi \mathrm{i}$ for an even perturbative potential $V$ as the one given in (1.2)). A straightforward bound of the Fourier coefficients of $L(t)$ reveals that $L(t)=$ constant + $\mathrm{O}\left(\exp \left(-\pi^{2} / h\right)\right)$ for real $t$, and from $(1.8)$, we can conclude that

$$
A=\varepsilon \int_{0}^{h / 2} M(t) \mathrm{d} t+\mathrm{O}\left(\varepsilon^{2}\right)=\varepsilon(L(h / 2)-L(0))+\mathrm{O}\left(\varepsilon^{2}\right)=\varepsilon \mathrm{O}\left(\exp \left(-\pi^{2} / h\right)\right)+\mathrm{O}\left(\varepsilon^{2}\right) .
$$

Thus, for fixed $h>0$, if $\varepsilon$ is small enough, the Melnikov term $\varepsilon(L(h / 2)-L(0))$, assumed to be not zero, is the dominant term for the formula of the area of the lobe. On the contrary, in the case that $h>0$ is a small parameter and $\varepsilon=h^{p}$-the case that we are interested in, according to the previous comments-, the Melnikov theory above described is not applicable, since it only gives the very coarse estimate $A=\mathrm{O}\left(h^{2 p}\right)$, and not the desired exponentially small behaviour of the splitting.

Our main result is that for $p>6$, the first term of formula (1.8) predicts correctly the exponentially small asymptotic behaviour of the lobe area, under a general condition on $V$.

Theorem 1.1 (Main Theorem) Let $\widehat{V}: \mathbb{C} \rightarrow \mathbb{C}$ be the Borel transform ${ }^{2}$ of the perturbative potential $V(y)$ given in (1.2), that is,

$$
\widehat{V}(\xi)=\sum_{n \geq 2} \frac{V_{n}}{(2 n-1) !} \xi^{2 n-1} .
$$

Assume that $\varepsilon=\mathrm{o}\left(h^{6} \ln ^{-1} h\right)$ and $h=\mathrm{o}(1)$. Then,

\footnotetext{
${ }^{2}$ We use the definition of Borel transform given in [SS96]: The Borel transform of $y^{k+1}$ is $\xi^{k} / k$ !, and it is extended by linearity.
} 
(a) If $\widehat{V}(2 \pi) \neq 0$, there exist exactly four primary homoclinic orbits (the reversible ones), and all the lobes have the same geometric area $|A|$, where

$$
A=8 \pi \widehat{V}(2 \pi) \varepsilon \mathrm{e}^{-\pi^{2} / h}\left[1+\mathrm{O}\left(h^{2}\right)\right] \quad\left(h \rightarrow 0^{+}\right) .
$$

(b) If $\widehat{V}(2 \pi)=0$, there may exist more primary homoclinic, but the area of any lobe is $\mathrm{O}\left(\varepsilon h^{2} \mathrm{e}^{-\pi^{2} / h}\right)$ when $h \rightarrow 0^{+}$.

Let us make some comments about this result.

The condition $\widehat{V}(2 \pi)=0$ is of codimension one, and therefore $\widehat{V}(2 \pi) \neq 0$ is a generic property. For instance, it holds for any non-zero polynomial $V$ with rational coefficients, since $\pi$ is a transcendental number. When it holds, the perturbed curves cross and the perturbed map is non-integrable [Cus78].

The hypotheses $V(y)$ even and $V^{\prime \prime}(0)=0$ are of technical character and have been assumed for the sake of simplicity and brevity. They are intended to preserve some properties of the unperturbed map. The first one preserves the symmetry $S(z)=-z$ (i.e., $F=S F S$ ), and allows us to give in proposition 2.2 a nice formula for the Melnikov function

$$
M(t)=8 \pi^{2} h^{-1} \mathrm{e}^{-\pi^{2} / h}\left[\widehat{V}(2 \pi) \sin (2 \pi t / h)+\mathrm{O}\left(h^{2}\right)\right], \quad t \in \mathbb{R} .
$$

When the perturbative potential $V(y)$ is not even, the splitting size in the first quadrant of figure 2 is also given in first order by the Melnikov approximation (1.13), but a different formula holds in the third quadrant.

The condition $V^{\prime \prime}(0)=0$ simply preserves the characteristic exponent $h$, since then the linear part of the map $F$ at the origin does not depend on the perturbation. If $V^{\prime \prime}(0)=V_{1} \neq 0$, one must change the unperturbed characteristic exponent $h$ by the perturbed one $\kappa=\kappa(h, \varepsilon)>0$ defined implicitly by $\cosh \kappa=\mu+V_{1} \varepsilon$ in all the above formulae and bounds. See [DR97b] for a numerical study of this fact.

For entire perturbations $V(y)$, the expression (1.12) is not a closed formula since $\widehat{V}(2 \pi)$ is given by a series. However, for a polynomial $V(y)$, the Melnikov function and the Melnikov potential can be explicitly expressed in terms of Jacobian elliptic functions [DR96].

The main difference of our work with respect to the previous exponentially small asymptotic formulae for other related problems is the character of the perturbation which is an arbitrary even entire function instead of a polynomial one as usual. This general perturbations can be handled since the unperturbed map that we consider is written in the same way as the separatrices that take place near resonances of integrable systems. In particular, the separatrix is contained in a neighbourhood $\mathrm{O}(h)$ of the origin, and the homoclinic solution $\xi^{0}(t)=\gamma \operatorname{sech} t$ is bounded up to a distance $\mathrm{O}(h)$ of its singularities. This is the key property that allows us to consider entire perturbations.

To finish these comments on the results, it is interesting to point out that exponentially small upper bounds of the splitting size can be obtained simply applying the results in [FS90, Fon95], giving rise to the following result. 
Theorem 1.2 (Upper Bound Theorem) Assume that $\varepsilon=\mathrm{O}\left(h^{p}\right)$ for some $p>0$. Then, given any $\alpha \in\left(0, \pi^{2}\right)$ there exists a constant $N=N(\alpha)$ such that the lobe area verifies

$$
A \leq N(\alpha) \mathrm{e}^{-\alpha / h} \quad\left(h \rightarrow 0^{+}\right)
$$

Of course, the conclusion of the Main Theorem is sharper than that of the Upper Bound Theorem since one does not have any control in the way that $N(\alpha)$ grows when $\alpha$ tends to $\pi^{2}$. However, the hypothesis $\varepsilon=\mathrm{O}\left(h^{p}\right)$, for some $p>0$, of the Upper Bound Theorem is weaker than the hypothesis $\varepsilon=\mathrm{o}\left(h^{6} \ln ^{-1} h\right)$ of the Main Theorem.

\section{The methodology}

It should be stressed that the problem considered here is easier than the one for the standard or Hénon maps. This has to do with the fact that in the example considered here, there appear in a natural way two parameters playing a very different rôle: a parameter $h$ which creates the hyperbolicity and the separatrix, but preserves the integrability of the maps, and another (perturbative) parameter $\varepsilon$ which gives rise to the splitting. In particular, an integrable map, defined on the whole plane, such that approximates well the perturbed map is easily obtained, whereas for the standard or Hénon maps this seems very difficult. This fact allows us to translate the main ideas of [DS92, DS97] to maps, since the rapidly forced systems there considered have a similar behaviour, i.e., they are close to integrable systems (see also [Gel93, Ang93]).

Let us notice that the proof of the exponentially small asymptotic formulae for rapidly forced systems follows easily once it is shown that the splitting is given in first order by the Melnikov function (a highly non-trivial fact). In [DS92] it is claimed that the problem for maps is more difficult than for flows, because "the linear difference equations that appear in that case do not lead to a computational Poincaré-Melnikov theory, as happens in the case of flows, and the analysis is more intricate" (sic). Recently, this obstacle has been overcome. In a previous work [DR96], the authors were able to develop a computational Melnikov theory for perturbations of an integrable planar map with a separatrix to a hyperbolic fixed point. Thus, the proof for rapidly forced systems can be adapted to the case of maps with two fundamental differences. First, difference equations (instead of differential equations) appear in the study of the invariant curves of the maps. Second, the Summation Formula given in [DR96] (instead of the Residue Theorem) must be used in the effective computation of the Melnikov function.

We are able to give a rigorous justification of the the Melnikov method by studying the perturbed invariant curves of the maps (1.1) for complex values of the discrete time $t$, as close as possible to the singularities of the unperturbed natural parameterization $z^{0}(t)$ given in (1.6). This approach was suggested by Lazutkin [Laz84] several years ago, for the case of the standard map.

Our measure of the splitting of the separatrices is given by the so-called splitting function $\Psi(s)$ defined in (2.22) as the graphic of the unstable curve over the stable one, in some "flow box" canonical coordinates. It is a $h$-periodic function, whose zeros 
give rise to homoclinic orbits, and all the splitting quantities can be obtained from it. For instance, the lobe area $A$ is the integral of the splitting function between two consecutive zeros. Another symplectic invariants used to measure the splitting, like the Lazutkin homoclinic invariant introduced in [GLT91] (which is simply the derivative of the splitting function at some of its zeros) or the area of the stochastic layer (see [Laz91, Tre96b]), can be computed. We have chosen the lobe area because it measures the flux along the homoclinic tangle, which is related to the study of transport [RW88, Mei92], and it has a clear geometric interpretation.

\section{Other applications}

As already said, the method used in this paper does not apply to the standard or Hénon maps, since we do not know which integrable map can play the rôle that is played here by the McMillan map. (Several unfruitful attempts have been done by the authors.) Nevertheless, this method is general enough and can be applied to many other situations.

As a first example, it can be applied to perturbations of other integrable area preserving maps like the standard-like maps given by Suris in [Sur89], or the twist maps associated to elliptic billiards. (The McMillan map is a particular case of the Suris maps.) For instance, the paper [LM96] is devoted to the study of a trigonometric Suris map and an exponentially small prediction for the lobe area is computed via the Melnikov function, which is likely to be proved by the tools developed here. Concerning perturbed elliptic billiards, they have been considered in several papers [LT93, Tab94, DR96, Lom96, Lev97], which contain exponentially small predictions for the splitting size, but there is still a lack of rigorous proof.

As a second application, we mention the study of the splitting of separatrices for some high-dimensional symplectic maps. A first step in this direction was contained in [DR97a], where exponentially small asymptotic predictions were computed via Melnikov methods for some perturbations of the McLachlan map (a high-dimensional generalization of the McMillan map). We hope that someday these heuristic formulae give rise to rigorous results in the same way as the heuristic formulae of [DR96] for the McMillan map have been the inspiration of this work.

As a last application, we consider the splitting of separatrices for volume preserving high-dimensional maps. In this case, the first results about exponentially small splitting of separatrices go back to [ACKR92]. We believe that some of the semi-analytical arguments contained in that paper can be rigorously developed following the ideas contained here, especially the part concerning the extension of the unstable curve. Of course, it will be necessary to consider maps depending on two parameters, as in our current example.

All these (and other related) topics are currently being researched. 


\section{Open issues and numerical tests}

The factor $\hat{V}(2 \pi)$ that appears in the asymptotic formula (1.12) looks amazing. At the present time, we do not know of an ultimate reason for its appearance (leaving aside the obvious fact that the computations lead to this factor). Resurgence tools in the same spirit as in [Laz93, HM93, Sur94, Sau95, Che95] seem suitable in order to get an insight into this factor.

Our estimates are valid only for $\varepsilon=\mathrm{o}\left(h^{6} \ln ^{-1} h\right)$, which is the condition required for the Extension Theorem 2.1 to hold on a complex rectangle $\mathcal{K}_{h}^{u}$ of values of the discrete time $t, \mathrm{O}(h)$-close to the singularities of the unperturbed natural parameterization $z^{0}(t)$ given in (1.6). Besides, our approach is not applicable on the complex rectangle $\mathcal{K}_{h}^{\mathrm{u}}$ for $\left|\varepsilon h^{-4}\right|$ big, as it is explained in remark 2.4. Consequently, it seems possible to improve slightly the hypothesis about the size of the perturbative parameter (i.e., $p>4$ ) without big changes of the method, whereas a proof of the optimal hypothesis (that is, $\varepsilon=\mathrm{o}(1)$ according to the numerical results described below), apparently requires a new approach (maybe a more accurate singularity analysis on a smaller complex rectangle [DMS97], or the continuous averaging method of D. Treschev [Tre97, Tre96a], or resurgence techniques).

There is numerical evidence that the parameters $h$ and $\varepsilon$ can be taken independent, with a $\mathrm{O}(\varepsilon)$ relative error if one changes slightly the asymptotic formula [DR97b]. Let us explain this. First, we note that the $\mathrm{O}\left(h^{2}\right)$ error term in the asymptotic formula (1.12) for the lobe area is generated by the $\mathrm{O}\left(h^{2}\right)$ error term in the asymptotic formula (1.13) for the Melnikov function. (There are higher order errors generated by other approximations.) Thus, we must compute more accurately the Melnikov function in order to improve the error. Let $\sum_{\ell \in \mathbb{Z}} v_{\ell}(h) \tau^{2 \ell}$ be the Laurent expansion around $\tau=0$ of the function $V(\gamma / \sin (h \tau))$. Lemma 4.2 implies that $A_{\text {theo }}:=\varepsilon(L(h / 2)-L(0))=\varepsilon \int_{0}^{h / 2} M(t) \mathrm{d} t$ is given by

$$
A_{\text {theo }}=8 \pi \varepsilon \sum_{k \geq 0} \frac{q^{2 k+1}}{1-q^{2(2 k+1)}} \widehat{v}_{2 k+1}(h)=8 \pi \varepsilon q\left[\widehat{v}_{1}(h)+\mathrm{O}\left(q^{2}\right)\right] \text {, }
$$

where $q=\mathrm{e}^{-\pi^{2} / h}$, and $\widehat{v}_{k}(h)=\sum_{\ell \geq 1}(2 \pi k)^{2 \ell-1} v_{-\ell}(h) /(2 \ell-1) !=\widehat{V}(2 \pi k)+\mathrm{O}\left(h^{2}\right) .\left(\widehat{v}_{k}(h)\right.$ are even entire functions of $h$, such that $\widehat{v}_{k}(0)=\widehat{V}(2 \pi k)$. If $V(y)$ is a polynomial, $\widehat{v}_{k}(h)$ can be computed in a finite number of steps.) Assuming that $\widehat{v}_{1}(0)=\widehat{V}(2 \pi) \neq 0$, numerical experiments lead us to the following conjecture:

$$
A=A_{\text {theo }}[1+\mathrm{O}(\varepsilon)], \quad A_{\text {theo }}=8 \pi \varepsilon \widehat{v}_{1}(h) \mathrm{e}^{-\pi^{2} / h}\left[1+\mathrm{O}\left(\mathrm{e}^{-2 \pi^{2} / h}\right)\right]
$$

where $h$ and $\varepsilon$ are independent small parameters, that is, they tend to zero in any independent way ${ }^{3}$. As an example of this conjecture, we take the cubic perturbation $V^{\prime}(y)=y^{3}$; then $\widehat{v}_{1}(h)=\pi \gamma^{4}\left[1+(\pi / h)^{2}\right] / 3 h^{2}$. The theoretical values $A_{\text {theo }}$ are shown

\footnotetext{
${ }^{3}$ When $\varepsilon$ is small but fixed and $h \rightarrow 0^{+}, A / A_{\text {theo }}$ tends to a constant $\Theta(\varepsilon)$, but $\Theta(\varepsilon)=1+\mathrm{O}(\varepsilon) \not \equiv 1$. Hence, the Melnikov method does not work for fixed $\varepsilon$.
} 
Table 1: Values of $A_{\text {theo }}, A_{\text {num }}$, and $\rho$, for $V^{\prime}(y)=y^{3}$.

\begin{tabular}{c|c|c|c|c}
$h$ & $\varepsilon$ & $A_{\text {theo }}$ & $A_{\text {num }}$ & $\rho$ \\
\hline $10^{-1}$ & $10^{-1}$ & $3.58720 \times 10^{-42}$ & $3.01433 \times 10^{-42}$ & -1.59696 \\
$10^{-2}$ & $10^{-2}$ & $6.06916 \times 10^{-429}$ & $5.98027 \times 10^{-429}$ & -1.46461 \\
$10^{-3}$ & $10^{-3}$ & $1.25846 \times 10^{-4287}$ & $1.25663 \times 10^{-4287}$ & -1.45182
\end{tabular}

in table 1 , versus the numerical computed ones $A_{\text {num }}$, together with the relative errors $\rho$ defined by $A_{\text {num }}=A_{\text {theo }}(1+\rho \varepsilon)$, for several values of the parameters $h$ and $\varepsilon$. See [DR97b] in order to learn how these fantastically small quantities can be numerically computed with a given arbitrary accuracy in a fast way. The key is to use a multipleprecision arithmetic and expand the local invariant curves to high order. To minimize the computer time it is necessary that once the parameters are given, the program itself decides: 1) the number of digits used in the arithmetic, and 2) the order used in the curve expansion.

\section{Organization of the paper}

The rest of the paper is devoted to the proof of the Main Theorem, except for the appendix where the proof of the Upper Bound Theorem is briefly sketched. In section 2, all the main ideas and tools are introduced. First, in the Flow Box Theorem it is stated that flow box coordinates can be constructed in a small neighbourhood of the local stable invariant curve. This is a quite standard result, and its proof has only been sketched. Next, in the Extension Theorem it is proved that the unstable curve can be prolonged until it enters into the neighbourhood where the flow box coordinates are defined. The proof of the Extension Theorem requires some original ideas, and it is deferred to section 3 to avoid a premature incursion into technicalities. Finally, the splitting function $\Psi$ is defined in the usual way, and its relationship with the Melnikov function is shown. All these results together give rise to the formulae that we are looking for.

\section{Proof of the Main Theorem}

For the sake of simplicity in the notation, the dependence on $h>0$ and $\varepsilon$ is usually not explicitly written, but it is always assumed to be analytic and bounded. (Otherwise it will be clearly stated.) In the sequel, $h$ will be a small enough quantity and $\varepsilon=$ $\mathrm{o}\left(h^{6} \ln ^{-1} h\right)$.

We first look for a local representation of the invariant curves associated to the origin $O=(0,0)$ of the map $F(z)$ given in (1.1). To such end, we will put the area preserving map $F(z)$ in normal form near the origin. We first normalize its linear part $\mathrm{d} F(O)$, which coincides with $\mathrm{d} F^{0}(O)$, due to the hypothesis $V^{\prime \prime}(0)=0$. 
From the expression (1.6) for $z^{0}(t)$ and $\xi^{0}(t)$, it follows that

$$
z^{0}(t)=2 \gamma \exp (\mp t)\left(\begin{array}{c}
\exp ( \pm h / 2) \\
\exp (\mp h / 2)
\end{array}\right)+\gamma \mathrm{O}(\exp (\mp 3 t)), \quad t \rightarrow \pm \infty
$$

so we introduce the linear change of variables (recall that $\lambda=\mathrm{e}^{h}$ )

$$
z=C \zeta, \quad C=2 \gamma\left(\begin{array}{cc}
\lambda^{-1 / 2} & \lambda^{1 / 2} \\
\lambda^{1 / 2} & \lambda^{-1 / 2}
\end{array}\right), \quad z=(x, y),
$$

which satisfies $C=\mathrm{O}(h)$ and $\operatorname{det} C=-8 \gamma^{3}$. This linear change conjugates the map $F(z)$ to an area preserving map $G(\zeta)=C^{-1} F(C \zeta)$ which is $\mathrm{O}(h)$-close to the identity:

$$
G(\zeta)=\left(\begin{array}{cc}
\lambda & 0 \\
0 & \lambda^{-1}
\end{array}\right) \zeta+h\left[\widehat{G}^{0}(\zeta, h)+\varepsilon \widehat{G}^{1}(\zeta, h)\right], \quad \widehat{G}^{0}, \widehat{G}^{1}=\mathrm{O}\left(\zeta^{3}\right) .
$$

The hypothesis $V^{\prime}(y)=\mathrm{O}\left(y^{3}\right)$ has been used to get the $\mathrm{O}\left(\zeta^{3}\right)$-behaviour for $\widehat{G}^{0}, \widehat{G}^{1}$. Moreover, since $V^{\prime}(y)$ is odd in $y, G(\zeta)$ is an odd analytic canonical map which has the origin as a hyperbolic fixed point, and therefore there exists an odd analytic canonical change of variables that conjugates $G$ to its Birkhoff normal form. This result is summarized in the following proposition.

Proposition 2.1 (Normal Form Theorem) There exists $\rho>0$ independent of $h$ and $\varepsilon$, and an odd canonical change of variables close to the identity map

$$
\zeta=\Phi(w)=\Phi^{0}(w)+\mathrm{O}(\varepsilon)=w+\mathrm{O}\left(w^{3}\right)
$$

real analytic on $B_{\rho}:=\left\{w \in \mathbb{C}^{2}:\|w\|<\rho\right\}$, such that conjugates the map $G(\zeta)$ to its Birkhoff normal form $N=\Phi^{-1} G \Phi$ given by

$$
N(w)=\left(u \mathrm{e}^{h \Lambda(I)}, v \mathrm{e}^{-h \Lambda(I)}\right), \quad w=(u, v), \quad I=u v,
$$

where

$$
\Lambda(I)=\Lambda^{0}(I)+\mathrm{O}(\varepsilon)=1+\mathrm{O}(I) .
$$

Proof. The existence of such canonical change of variables is a celebrated result due to J. Moser [Mos56]. (See also [Del95] for a modern and more direct treatment.) For families of maps close to the identity, the neighbourhood of the origin on which the change $\Phi(w)$ and the normal form $N(w)$ are convergent is independent of the parameters [FS90, Fon95]. It is also a well-known fact that the change and the normal form depend analytically on the parameters, so the perturbed objects are the unperturbed ones plus $\mathrm{O}(\varepsilon)$ (see [DGJS97] for a complete proof in the case of Hamiltonian flows). Finally, the change is odd because the normal form and the original map are odd.

The variables $w=(u, v)$ are called normal coordinates. As $I=u v$ (or equivalently $\Lambda(I)$ ) is a first integral of the Birkhoff normal $N$, the orbit inside $B_{\rho}$ of every point $w=$ $(u, v)$ can be explicitly written: $N^{k}(w)=\left(u \mathrm{e}^{k h \Lambda(I)}, v \mathrm{e}^{-k h \Lambda(I)}\right)$. In particular, natural parameterizations for the local invariant curves $\mathcal{W}_{\text {loc }}^{\mathrm{u}, \mathrm{s}}$ of the map $F$ can be immediately obtained, satisfying also several properties that are listed in the next corollary. 
Corollary 2.1 (Local Invariant Curves) Let $T>0$ be a constant independent of $h$ and $\varepsilon$ such that $T>-2 \ln \rho$, where $\rho>0$ is the constant given in the previous proposition. Let $\mathcal{J}^{\mathrm{u}, \mathrm{s}}$ be the vertical half planes

$$
\mathcal{J}^{\mathrm{u}}=\{t \in \mathbb{C}: \Re t \leq-T / 2\}, \quad \mathcal{J}^{\mathrm{s}}=\{t \in \mathbb{C}: \Re t \geq T / 2\} .
$$

Then, there exist analytic parameterizations $z^{\mathrm{u}, \mathrm{s}}: \mathcal{J}^{\mathrm{u}, \mathrm{s}} \rightarrow \mathcal{W}_{\mathrm{loc}}^{\mathrm{u}, \mathrm{s}}$, such that

(a) they are natural parameterizations: $F\left(z^{\mathrm{u}, \mathrm{s}}(t)\right)=z^{\mathrm{u}, \mathrm{s}}(t+h)$.

Moreover, they are uniquely determined by the following properties:

(b) For $\varepsilon=0, z^{\mathrm{u}, \mathrm{s}}(t)$ coincide with the natural parameterization $z^{0}(t)$ of the unperturbed separatrix given in (1.6). For $\varepsilon \neq 0$, they satisfy the estimates

$$
\begin{aligned}
& z^{\mathrm{u}}(t)-z^{0}(t)=\mathrm{O}\left(\varepsilon h \mathrm{e}^{3 \Re t}\right), \forall t \in \mathcal{J}^{\mathrm{u}}, \\
& z^{\mathrm{s}}(t)-z^{0}(t)=\mathrm{O}\left(\varepsilon h \mathrm{e}^{-3 \Re t}\right), \quad \forall t \in \mathcal{J}^{\mathrm{s}} .
\end{aligned}
$$

(c) They are $\pi \mathrm{i}$-antiperiodic, that is, $z^{\mathrm{u}, \mathrm{s}}(t+\pi \mathrm{i})=-z^{\mathrm{u}, \mathrm{s}}(t)$.

Proof. First, we will prove the uniqueness of solutions. Assume that $z^{\mathrm{u}, \mathrm{s}}(t)$ are analytic functions verifying properties (a)-(c). Let $\mathcal{V}=\left\{r \in \mathbb{C}:|r| \leq \mathrm{e}^{-T / 2}\right\}$. By property (c), $z^{\mathrm{u}, \mathrm{s}}(t)$ are $2 \pi \mathrm{i}$-periodic, so they can be written in terms of the variable $r=\mathrm{e}^{t}$, giving rise to the analytic functions $\sigma^{\mathrm{u}, \mathrm{s}}: \mathcal{V} \rightarrow \mathcal{W}_{\text {loc }}^{\mathrm{u}, \mathrm{s}}$ implicitly defined by $\sigma^{\mathrm{u}}\left(\mathrm{e}^{t}\right)=z^{\mathrm{u}}(t)$, and $\sigma^{\mathrm{s}}\left(\mathrm{e}^{-t}\right)=z^{\mathrm{s}}(t)$. Now, property (a) implies that

$$
F\left(\sigma^{\mathrm{u}}(r)\right)=\sigma^{\mathrm{u}}(\lambda r), \quad F\left(\sigma^{\mathrm{s}}(r)\right)=\sigma^{\mathrm{s}}\left(\lambda^{-1} r\right)
$$

whereas property (b) gives rise to

$$
\sigma^{\mathrm{u}, \mathrm{s}}(0)=\left(\begin{array}{l}
0 \\
0
\end{array}\right), \quad \frac{\mathrm{d} \sigma^{\mathrm{u}}}{\mathrm{d} r}(0)=2 \gamma\left(\begin{array}{c}
\lambda^{-1 / 2} \\
\lambda^{1 / 2}
\end{array}\right), \quad \frac{\mathrm{d} \sigma^{\mathrm{s}}}{\mathrm{d} r}(0)=2 \gamma\left(\begin{array}{c}
\lambda^{1 / 2} \\
\lambda^{-1 / 2}
\end{array}\right) .
$$

A direct computation using Taylor series shows that equations (2.7)-(2.8) have unique formal solutions, so we have proved the uniqueness of $\sigma^{\mathrm{u}, \mathrm{s}}$, and hence, the uniqueness of $z^{\mathrm{u}, \mathrm{s}}$.

To end, we will check that the functions defined by $z^{\mathrm{u}, \mathrm{s}}(t)=C \Phi\left(w^{\mathrm{u}, \mathrm{s}}(t)\right)$, for $t \in \mathcal{J}^{\mathrm{u}, \mathrm{s}}$, where $w^{\mathrm{u}}(t)=\left(\mathrm{e}^{t}, 0\right)$, and $w^{\mathrm{s}}(t)=\left(0, \mathrm{e}^{-t}\right)$, verify all the required properties.

First, we note that hypothesis $T>-2 \ln \rho$ implies $\mathrm{e}^{t} \in B_{\rho}$ for $t \in \mathcal{J}^{\mathrm{u}}$, and $\mathrm{e}^{-t} \in B_{\rho}$ for $t \in \mathcal{J}^{\mathrm{s}}$, so $z^{\mathrm{u}, \mathrm{s}}(t)$ are well-defined and analytic on $\mathcal{J}^{\mathrm{u}, \mathrm{s}}$. From equations (2.3)(2.4) we get $N\left(w^{\mathrm{u}, \mathrm{s}}(t)\right)=w^{\mathrm{u}, \mathrm{s}}(t+h)$, and property (a) can be deduced by means of the change $z=C \Phi(w)$. The coincidence of $z^{\mathrm{u}, \mathrm{s}}(t)$ and $z^{0}(t)$, for $\varepsilon=0$, is due to the uniqueness of solutions in the unperturbed problem. Formulae (2.5)-(2.6) are a consequence of approximation (2.2), the estimate $C=\mathrm{O}(h)$, the definitions of $z^{\mathrm{u}, \mathrm{s}}(t)$, and the equalities $z^{0}(t)=C \Phi^{0}\left(w^{\mathrm{u}, \mathrm{s}}(t)\right)$, for $t \in \mathcal{J}^{\mathrm{u}, \mathrm{s}}$. This proves property (b). Finally, property (c) follows from the fact that $\Phi(w)$ is odd in $w$. 
Remark 2.1 We have taken advantage of the hypotheses $V^{\prime}(y)$ odd, $V^{\prime}(y)=\mathrm{O}\left(y^{3}\right)$, to obtain $\pi$ i-antiperiodic parameterizations, $\mathrm{O}\left(\mathrm{e}^{3 \Re t}\right)$-close to the separatrix in the asymptotic expressions $(2.5),(2.6)$, instead of the standard $2 \pi$ i-periodic parameterizations, $\mathrm{O}\left(\mathrm{e}^{2 \Re t}\right)$-close to the separatrix.

Now, we are ready to state (and prove) a Flow Box Theorem outside of the local unstable invariant curve, as a direct consequence of the Birkhoff normal form. (It can be proved without using it [Gel96], a fact that can be important for high-dimensional situations, since the Birkhoff normal form is usually divergent for such cases.)

Corollary 2.2 (Flow Box Theorem) Let $B_{\rho}$ be the ball where the normal coordinates are defined, and $U=C \Phi(\mathcal{U})$ with $\mathcal{U}=\left\{w=(u, v) \in B_{\rho}, \Re v>0\right\}$. Then, there exists an analytic canonical change of variables

$$
z \in U \longmapsto(s, e)=P(z)=(S(z), E(z)),
$$

such that conjugates the map (1.1) to the flow box map $P F P^{-1}$ given by

$$
s \mapsto s+h, \quad e \mapsto e .
$$

Besides, the change verifies the following properties:

(a) The perturbed change is close to the unperturbed one, more precisely

$$
S(z)=S^{0}(z)+\mathrm{O}(\varepsilon), \quad E(z)=E^{0}(z)+\mathrm{O}\left(\varepsilon h^{3}\right) .
$$

(b) Along the local stable curve $z^{\mathrm{s}}(t)$, the flow box functions (2.9) satisfy

$$
S\left(z^{\mathrm{s}}(t)\right)=t, \quad E\left(z^{\mathrm{s}}(t)\right)=0 .
$$

(c) The function $E^{0}: U \rightarrow \mathbb{C}$ is a first integral of the unperturbed map $F^{0}$ such that

$$
E^{0}(z)=H^{0}(z)+\mathrm{O}\left(\left(H^{0}(z)\right)^{2}\right)
$$

where $H^{0}: \mathbb{C}^{2} \rightarrow \mathbb{C}$ is the polynomial first integral given in (1.5).

Proof. Let $\Theta(I)$ be the function determined by the conditions $\Theta^{\prime}(I)=\Lambda(I), \Theta(0)=0$. Then, for any $\beta \neq 0$, the analytic change of variables

$$
w=(u, v) \in \mathcal{U} \mapsto(s, e)=\mathcal{P}(w)=(\mathcal{S}(w), \mathcal{E}(w))=\left(-\frac{\log v}{\Theta^{\prime}(I)}, \beta \Theta(I)\right), \quad I=u v
$$

conjugates the normal form (2.3) to the flow box map (2.10), and possesses constant Jacobian $\operatorname{det}[\mathrm{d} \mathcal{P}(w)] \equiv \beta$.

Now, the change (2.9) is defined as the composition of $(s, e)=\mathcal{P}(w)$ with the inverse of $z=C \Phi(w)$, that is, taking $P=\mathcal{P} \Phi^{-1} C^{-1}, E(z)=\mathcal{E}(w)$, and $S(z)=\mathcal{S}(w)$. We 
also choose $\beta=\operatorname{det} C=-8 \gamma^{3}$ and then, by construction, $(s, e)=P(z)$ is defined on the open $U=C \Phi(\mathcal{U})$, conjugates the map (1.1) in the flow box map (2.10), and is an analytic canonical change of variables.

Property (a) follows from equation (2.4), the definition of $\Theta(I)$, and the estimate $\beta=-8 \gamma^{3}=\mathrm{O}\left(h^{3}\right)$. Property (b) is a direct consequence of the formulae $\mathcal{S}\left(0, \mathrm{e}^{-t}\right)=t$, and $\mathcal{E}\left(0, \mathrm{e}^{-t}\right)=0$, together with the definition $z^{\mathrm{s}}(t)=C \Phi\left(0, \mathrm{e}^{-t}\right)$, for $t \in \mathcal{J}^{\mathrm{s}}$, introduced in the proof of the previous corollary. In order to prove (c), we note that $z=C w$ implies the identity $\beta I=\left(x^{2}-2 \mu x y+y^{2}\right) / 2 \gamma$, where $z=(x, y), w=(u, v)$, and $I=u v$, as usual. Now, using that $z=C \Phi(w)=C w+\mathrm{O}\left(w^{3}\right)$, we deduce that

$$
E^{0}(z)=\mathcal{E}^{0}(w)=\beta \Theta^{0}(I)=\beta I+\mathrm{O}\left(I^{2}\right)=\left(x^{2}-2 \mu x y+y^{2}\right) / 2 \gamma+\mathrm{O}\left(z^{4}\right),
$$

that is, the quadratic parts at the origin of the first integrals $H^{0}(z), E^{0}(z)$ coincide. Moreover, $H^{0}, E^{0}$ are functionally dependent maps, since in the normal coordinates $w=(u, v)$ they become functions of a single variable: $I=u v$. In particular, the reasonings above imply that they take the form $\beta I+\mathrm{O}\left(I^{2}\right)$. Hence, there exists an analytic function $\kappa$ defined on a neighbourhood of $0 \in \mathbb{C}$, such that $E^{0}(z)=\kappa\left(H^{0}(z)\right)$, $\kappa(0)=0$, and $\kappa^{\prime}(0)=1$. This proves $(\mathrm{c})$.

Remark 2.2 A more detailed (but unnecessary for present purposes) study shows that $H^{0}(z), E^{0}(z)$ do not coincide: $E^{0}(z)=\kappa\left(H^{0}(z)\right)$, but $\kappa$ is not the identity. This is in contrast with what happens in the continuous case, in which the unperturbed first integral constructed via the Flow Box Theorem coincides with the initial unperturbed Hamiltonian [DS97]. Equation (2.13) is intended to overcome this difficulty; it shows that $H^{0}(z), E^{0}(z)$ coincide in the first approximation near the unperturbed separatrix, where both functions vanish.

Once we have at our disposal of a good local framework, we need some global knowledge of the invariant curves. In particular, we want to extend the unstable invariant curve until it enters into the open set $U$ where the flow box coordinates are defined. Roughly speaking, in order to show that the Melnikov method gives the correct behaviour of the splitting size, it is necessary to see that the perturbed invariant curves are close to the unperturbed separatrix not only for real $t$, but also for complex $t$. This is not trivial, because the function $z^{0}(t)$ given by (1.6) has poles on the complex field. The closest poles to the real line have imaginary part $\pm \pi / 2$, so we will not be able to control the growth of the parameterization $z^{\mathrm{u}}(t)$ for $\Im t= \pm \pi / 2$. Following [DS92], we will restrict ourselves to a complex rectangle of imaginary width equal to $\pi / 2-h$. The final result is summarized in the following theorem.

Theorem 2.1 (Extension Theorem) If $h=\mathrm{o}(1)$ and $\varepsilon=\mathrm{o}\left(h^{6} \ln ^{-1} h\right)$, the natural parameterization $z^{\mathrm{u}}(t)$ can be analytically extended to the complex rectangle

$$
\mathcal{K}_{h}^{\mathrm{u}}=\{t \in \mathbb{C}:|\Im t| \leq \pi / 2-h,|\Re t| \leq T\} .
$$

In addition, the following estimate holds

$$
z^{\mathrm{u}}(t)-z^{0}(t)=\mathrm{O}\left(\varepsilon h^{-3}\right), \quad t \in \mathcal{K}_{h}^{\mathrm{u}} .
$$


The proof of this theorem involves several technicalities, such as a good choice of the solutions of the variational linear difference equations associated to the separatrix, and a suitable space on which to formulate a fixed point equation that allows us to give quantitative results, using some contraction argument. It is deferred to section 3, jointly with a discussion in remark 3.2 about the numerical experiments performed to check the optimality of the Extension Theorem. Here, we only want to state that we have checked numerically that: i) bound (2.14) is optimal on $\mathcal{K}_{h}^{\mathrm{u}}$, ii) it holds under the weaker conditions $h$ small, $\varepsilon=\mathrm{o}\left(h^{3}\right)$.

Remark 2.3 It has to be noted here that far from the singularities the Extension Theorem can be improved. Let $\delta \in(0, \pi / 2]$ be fixed. Then, if $h$ and $|\varepsilon|$ are small enough, the following estimate holds

$$
z^{\mathrm{u}}(t)-z^{0}(t)=\mathrm{O}(\varepsilon h), \quad t \in \mathcal{K}_{\delta}^{\mathrm{u}} .
$$

See remark 3.3 for a proof. The difficulty (and the relevance) of the previous theorem is to reach a distance $\mathrm{O}(h)$ of the singularities.

The unperturbed natural parameterization $z^{0}(t)$ arrives and stays at the open set $U$ for $\Re t \geq T / 2$ and $|\Im t|<\pi / 2$. By estimate (2.14), the same happens to $z^{\mathrm{u}}(t)$ for $T / 2 \leq \Re t \leq T$ and $|\Im t| \leq \pi / 2-h$. On $U$, the flow box functions (2.9) are defined, and therefore they can be evaluated on the unstable curve giving rise to the functions

$$
S^{\mathrm{u}}(t):=S\left(z^{\mathrm{u}}(t)\right), \quad E^{\mathrm{u}}(t):=E\left(z^{\mathrm{u}}(t)\right) .
$$

Using that $z^{\mathrm{u}}(t)$ is an analytic natural parameterization and equation (2.10), it turns out that $S^{\mathrm{u}}(t)-t$ and $E^{\mathrm{u}}(t)$ are $h$-periodic analytic functions, defined for $T / 2 \leq \Re t \leq T$ and $|\Im t| \leq \pi / 2-h$. By periodicity, they can be considered defined on the whole complex strip

$$
\mathcal{I}_{h}=\{t \in \mathbb{C}:|\Im t| \leq \pi / 2-h\}
$$

as well as $S^{\mathrm{u}}(t)$.

Next proposition collects several computations about the Melnikov function $M(t)$ and the functions $S^{\mathrm{u}}(t), E^{\mathrm{u}}(t)$. In particular, it is proved the crucial fact that $E^{\mathrm{u}}(t)$ is given in first order by the Melnikov approximation $\varepsilon M(t)$, at least on the complex strip $\mathcal{I}_{h}$. Since both functions are $h$-periodic, the difference between $E^{\mathrm{u}}(t)-E_{0}^{\mathrm{u}}$ and $\varepsilon M(t)$ is proved to be $\mathrm{O}\left(\mathrm{e}^{-\pi^{2} / h}\right)$ for real $t$, where $E_{0}^{\mathrm{u}}$ is the zero order Fourier coefficient of $E^{\mathrm{u}}(t)$. Moreover, the asymptotic behaviour of the Melnikov function and its derivative is explicitly computed and, finally, the equation $s=S^{\mathrm{u}}(t)$ is inverted on the real line, preparing the way for the definition of the splitting function.

Proposition 2.2 With the previous notations, definitions and assumptions:

(a) The following estimates hold

$$
\begin{array}{ll}
E^{\mathrm{u}}(t)=\varepsilon M(t)+\mathrm{O}\left(\varepsilon h^{3}, \varepsilon^{2} h^{-5}\right), & \forall t \in \mathcal{I}_{h}, \\
E^{\mathrm{u}}(t)=E_{0}^{\mathrm{u}}+\varepsilon M(t)+\mathrm{O}\left(\varepsilon h^{3}, \varepsilon^{2} h^{-5}\right) \mathrm{e}^{-\pi^{2} / h}, & \forall t \in \mathbb{R},
\end{array}
$$


where $E_{0}^{\mathrm{u}}=h^{-1} \int_{0}^{h} E^{\mathrm{u}}(t) \mathrm{d} t, M(t)$ is the Melnikov function (1.9), and $\mathcal{I}_{h}$ is the complex strip (2.17).

(b) The equation $s=S^{\mathrm{u}}(t)$ can be inverted for real $t$. Denoting $t=s^{\mathrm{u}}(s)$ its inverse, the function $\phi^{\mathrm{u}}(s)=s^{\mathrm{u}}(s)-s$ is $\mathrm{O}(\varepsilon)$, analytic, and $h$-periodic.

(c) Let $M(t)$ be the Melnikov function as defined in (1.10). Then,

$$
\begin{aligned}
& M(t)=8 \pi^{2} h^{-1} \mathrm{e}^{-\pi^{2} / h}\left[\widehat{V}(2 \pi) \sin (2 \pi t / h)+\mathrm{O}\left(h^{2}\right)\right], t \in \mathbb{R}, \\
& M^{\prime}(t)=16 \pi^{3} h^{-2} \mathrm{e}^{-\pi^{2} / h}\left[\widehat{V}(2 \pi) \cos (2 \pi t / h)+\mathrm{O}\left(h^{2}\right)\right], \quad t \in \mathbb{R} .
\end{aligned}
$$

The proof of this proposition is somewhat technical, and is deferred to section 4 . In particular, the proof of the asymptotic behaviour of the Melnikov function relies strongly on the theory of elliptic functions.

Remark 2.4 The function $E(z)$ given in $(2.9)$ is $\mathrm{O}\left(h^{3}\right)$ on $U$, due to the factor $\beta=$ $\operatorname{det} C=-8 \gamma^{3}=\mathrm{O}\left(h^{3}\right)$ that appears during the proof of corollary 2.2. Hence, $E^{\mathrm{u}}(t)=$ $E\left(z^{\mathrm{u}}(t)\right)$ is $\mathrm{O}\left(h^{3}\right)$ on the complex strip $\mathcal{I}_{h}$ given in (2.17). Besides, in lemma 4.3, it is stated that the $k$-th order Fourier coefficient of the Melnikov function $M(t)$ is $\mathrm{O}\left(h^{-1} k \exp \left(-2 \pi|k| r_{h} / h\right)\right)$, where $r_{h}=\pi / 2-h$. This proves that $M(t)$ is $\mathrm{O}\left(h^{-1}\right)$ on the complex strip $\mathcal{I}_{h}$, since it has zero mean. For $\left|\varepsilon h^{-4}\right|$ big, both estimates imply that on the complex strip $\mathcal{I}_{h}$, the dominant term of $E^{\mathrm{u}}(t)$ is not $\varepsilon M(t)$.

Equations (2.12) and (2.16) imply that the local stable curve $z^{\mathrm{s}}(t)$ and the arriving unstable one $z^{\mathrm{u}}(t)$ in the flow box coordinates can be written in parametric form as

$$
(s, e)=(S, E)\left(z^{\mathrm{s}}(t)\right)=(t, 0), \quad(s, e)=(S, E)\left(z^{\mathrm{u}}(t)\right)=\left(S^{\mathrm{u}}(t), E^{\mathrm{u}}(t)\right) .
$$

Therefore, it is very natural to introduce the splitting function $\Psi$ given implicitly by $\Psi\left(S^{\mathrm{u}}(t)\right)=E^{\mathrm{u}}(t)$. According to proposition 2.2, the function $S^{\mathrm{u}}$ is invertible on the real line, so the splitting function can be given explicitly by

$$
\Psi(s)=E^{\mathrm{u}}\left(s^{\mathrm{u}}(s)\right)=E^{\mathrm{u}}\left(s+\phi^{\mathrm{u}}(s)\right), \quad s \in \mathbb{R} .
$$

Then, the arriving unstable curve and the local stable one can be written as

$$
e=e^{\mathrm{u}}(s)=\Psi(s), \quad e=e^{\mathrm{s}}(s)=0,
$$

in the system of flow box coordinates $(s, e)$. The relation between the splitting function $\Psi(s)$ and the splitting size is shown in the next proposition.

Proposition 2.3 The splitting function is a h-periodic real analytic function such that:

(a) Its real zeros correspond to the primary homoclinic points. 
(b) Its integral between two consecutive real zeros is equal to the (algebraic) area of the lobe between the corresponding primary homoclinic points.

(c) It has zero mean, that is, $\Psi_{0}=h^{-1} \int_{0}^{h} \Psi(s) \mathrm{d} s=0$.

(d) For $s \in \mathbb{R}$, the following asymptotic estimate holds

$$
\Psi(s)=8 \pi^{2} h^{-1} \varepsilon \mathrm{e}^{-\pi^{2} / h}\left[\widehat{V}(2 \pi) \sin (2 \pi s / h)+\mathrm{O}\left(h^{2}, \varepsilon h^{-4}\right)\right] .
$$

Proof. The splitting function (2.22) is analytic $h$-periodic because so are $E^{\mathrm{u}}$ and $\phi^{\mathrm{u}}$.

(a) is direct from equation (2.23).

(b) also follows from (2.23), since the flow box coordinates $(s, e)$ are canonical.

(c) is a consequence of the fact that the area of the inner lobes and the outer ones coincide. This can be easily seen making one iterate of the map $F: \mathbb{R}^{2} \rightarrow \mathbb{R}^{2}$ and using that the total area between the global invariant curves is invariant.

(d) Equation (2.21) implies that $M^{\prime}(s)=\mathrm{O}\left(h^{-2} \mathrm{e}^{-\pi^{2} / h}\right)$, for $s \in \mathbb{R}$, and applying Taylor's Theorem to $M\left(s^{\mathrm{u}}(s)\right)=M(s+\mathrm{O}(\varepsilon))$ we get

$$
M\left(s^{\mathrm{u}}(s)\right)=M(s)+\mathrm{O}\left(\varepsilon h^{-2} \mathrm{e}^{-\pi^{2} / h}\right), \quad s \in \mathbb{R} .
$$

By the definition (2.22) of $\Psi(s)$, and applying (2.19), we have for real $s$

$$
\Psi(s)=E_{0}^{\mathrm{u}}+\varepsilon M\left(s^{\mathrm{u}}(s)\right)+\mathrm{O}\left(\varepsilon h^{3}, \varepsilon^{2} h^{-5}\right) \mathrm{e}^{-\pi^{2} / h}=E_{0}^{\mathrm{u}}+\varepsilon M(s)+\mathrm{O}\left(\varepsilon h^{3}, \varepsilon^{2} h^{-5}\right) \mathrm{e}^{-\pi^{2} / h} .
$$

Finally, since the splitting function and the Melnikov function have zero mean, we obtain that $E_{0}^{\mathrm{u}}=\mathrm{O}\left(\varepsilon h^{3}, \varepsilon^{2} h^{-5}\right) \mathrm{e}^{-\pi^{2} / h}$, and (2.24) follows from (2.20).

Now, the Main Theorem can be trivially deduced from the previous proposition, and especially from the asymptotic estimate given in (2.24). Note that $|\varepsilon| h^{-4} \ll h^{2}$, because we are assuming $\varepsilon=\mathrm{o}\left(h^{6} \ln ^{-1} h\right)$.

\section{Proof of the Extension Theorem}

\subsection{A simplification}

Let $\mathcal{J}$ be the vertical half plane $\mathcal{J}=\{t \in \mathbb{C}: \Re t \leq-T\}$. Let us recall that $F(x, y)=$ $(y,-x+f(y))$, where $f(y)=f^{0}(y)+\varepsilon V^{\prime}(y)$, and $f^{0}(y)=2 \mu y /\left(1+y^{2}\right)$. Thus, using the property of natural parameterization $F\left(z^{\mathrm{u}}(t)\right)=z^{\mathrm{u}}(t+h)$, there exists a unique analytic function $\xi: \mathcal{J} \rightarrow \mathbb{C}$ such that $z^{\mathrm{u}}(t)$ can be written in the form

$$
\left.z^{\mathrm{u}}(t)=(\xi(t-h / 2), \xi(t+h / 2))\right), \quad \forall t \in \mathcal{J}
$$

From corollary 2.1 we deduce that $\xi(t)$ is the unique analytic function verifying the following properties: (a) $\xi(t+h)+\xi(t-h)=f(\xi(t))$, (b) $\sup _{\mathcal{J}}\left\{\mathrm{e}^{-3 \Re t}\left|\xi(t)-\xi^{0}(t)\right|\right\}<\infty$, and (c) $\xi$ is $\pi$ i-antiperiodic. 
To compare the perturbed solution $\xi(t)$ with the known unperturbed one $\xi^{0}(t)$ given in (1.6) we introduce their difference $\eta(t)=\xi(t)-\xi^{0}(t)$, as well as the linear operator $\eta \mapsto L \eta$ defined by

$$
(L \eta)(t)=\eta(t+h)-\left(f^{0}\right)^{\prime}\left(\xi^{0}(t)\right) \eta(t)+\eta(t-h), \quad f^{0}(y)=\frac{2 \mu y}{1+y^{2}},
$$

and the nonlinear one $\eta \mapsto \mathcal{T} \eta$ given by

$$
(\mathcal{T} \eta)(t)=\Phi(t, \eta(t)), \quad \Phi(t, \eta)=\Phi^{0}(t, \eta)+\varepsilon \Phi^{1}(t, \eta)
$$

where

$$
\Phi^{0}(t, \eta)=f^{0}\left(\xi^{0}(t)+\eta\right)-f^{0}\left(\xi^{0}(t)\right)-\left(f^{0}\right)^{\prime}\left(\xi^{0}(t)\right) \eta, \quad \Phi^{1}(t, \eta)=V^{\prime}\left(\xi^{0}(t)+\eta\right) .
$$

Then, $\eta: \mathcal{J} \rightarrow \mathbb{C}$ is the unique analytic function verifying the following properties: (a') $L \eta=\mathcal{T} \eta$, (b') $\sup _{\mathcal{J}}\left\{\mathrm{e}^{-3 \Re t}|\eta(t)|\right\}<\infty$, and (c') $\eta$ is $\pi$ i-antiperiodic.

The idea of the proof is to invert the linear operator $L$ and solve the resulting fixed point equation $\eta=L^{-1} \mathcal{T} \eta$ in a suitable Banach space using some contraction argument. Properties (b')-(c'), jointly with that fact that $\xi^{0}(t)$ has a pole on $t=\pi \mathrm{i} / 2$, lead us naturally to introduce the following complex Banach space

$$
\mathcal{X}_{\delta}=\left\{\begin{array}{ll}
\phi: \mathcal{D}_{\delta} \rightarrow \mathbb{C} & \text { continuous on } \mathcal{D}_{\delta} \text { and analytic on its interior, } \\
& \pi \text { i-antiperiodic such that }\|\phi\|_{\delta}<\infty
\end{array}\right\},
$$

where $\|\phi\|_{\delta}=\sup _{\mathcal{D}_{\delta}}\left\{\mathrm{e}^{-3 \Re t}|\phi(t)|\right\}$, and $\mathcal{D}_{\delta}$ is the union of the vertical half plane $\mathcal{J}$ and a complex rectangle $\mathcal{K}_{\delta}$

$$
\mathcal{D}_{\delta}=\mathcal{J} \cup \mathcal{K}_{\delta}, \quad \mathcal{K}_{\delta}=\{t \in \mathbb{C}:|\Re t| \leq T,|\Im t| \leq \pi / 2-\delta\}
$$

Now, from the relations between the natural parameterizations $z^{0}(t), z^{\mathrm{u}}(t)$ and the functions $\xi^{0}(t), \xi(t)$ given in equations (1.6), (3.1), it follows that the Extension Theorem is a corollary of the next result on the function $\eta(t)=\xi(t)-\xi^{0}(t)$.

Proposition 3.1 If $h$ is small enough and $\varepsilon=\mathrm{o}\left(h^{6} \ln ^{-1} h\right)$, there exists a unique $\eta^{*} \in$ $\mathcal{X}_{h}$ verifying the nonlinear difference equation $L \eta=\mathcal{T} \eta$ such that

$$
\left\|\eta^{*}\right\|_{h}=\mathrm{O}\left(\varepsilon h^{-3}\right)
$$

The rest of this section is devoted to prove proposition 3.1 .

\subsection{The linear equation}

First, we present the method of solution of the linear non-homogeneous difference equation associated to the operator $L$ introduced in (3.2). The tools developed here are inspired by the ones contained in the papers [Tab94, Gel96]. In fact, we have adapted 
the general setting of [Gel96] to our concrete problem, where once a fundamental set of solutions of the homogeneous equation is known, the method of variation of parameters is applied to solve the non-homogeneous one.

Following this scheme, we consider first the homogeneous equation

$$
L \eta=0 .
$$

It is worth noting that (3.7) is the variational difference equation associated to the unperturbed homoclinic solution $\xi^{0}(t)$, see (1.7). Thus, a solution of this variational equation is simply the derivative of $\xi^{0}(t)$. Another independent solution can be also computed, as described in the following lemma, whose proof is straightforward. (It is important to stress that we will be able to prove proposition 3.1, due to our explicit knowledge of a fundamental set of solutions of the homogeneous equation.)

Lemma 3.1 The general solution of the homogeneous equation (3.7) has the form

$$
\eta_{\mathrm{h}}(t)=\alpha_{1}(t) \eta_{1}(t)+\alpha_{2}(t) \eta_{2}(t),
$$

where $\alpha_{1}(t), \alpha_{2}(t)$ are arbitrary $h$-periodic functions,

$$
\eta_{1}(t)=\left(\xi^{0}\right)^{\prime}(t)=-\gamma \frac{\tanh t}{\cosh t}
$$

and

$$
\eta_{2}(t)=\frac{A_{1}+A_{2} \sinh ^{2} t+A_{3}(t-c) \tanh t}{\gamma^{2} \cosh t}, \quad A_{1}=\mu^{2}, A_{2}=-\frac{1}{2}, A_{3}=-\frac{3 \gamma \mu}{2 h},
$$

$c \in \mathbb{C}$ being an arbitrary constant.

Moreover, $W(t) \equiv 1$, where $W(t)=W\left[\eta_{1}, \eta_{2}\right](t)$ denotes the Wronskian of the fundamental set of solutions $\left\{\eta_{1}, \eta_{2}\right\}$, that is,

$$
W(t)=\eta_{1}(t) \eta_{2}(t+h)-\eta_{1}(t+h) \eta_{2}(t)=\eta_{1}(t) \Delta \eta_{2}(t)-\eta_{2}(t) \Delta \eta_{1}(t),
$$

where $\Delta \eta(t)=\eta(t+h)-\eta(t)$.

Remark 3.1 A change of the free constant $c \in \mathbb{C}$ only changes $\eta_{2}(t)$ by adding a multiple of $\eta_{1}(t)$ to it. We will take advantage of this freedom to get a second solution $\eta_{2}(t)$ as regular as possible on its poles $t_{\mathrm{p}}^{ \pm}= \pm \pi \mathrm{i} / 2$. For instance, setting $c= \pm \pi \mathrm{i} / 2$, $t_{\mathrm{p}}^{ \pm}$becomes a simple pole of $\eta_{2}(t)$, instead of a double one. This choice of $c$ allows us to assume only the number "6" in the exponent of $\varepsilon=\mathrm{o}\left(h^{6} \ln ^{-1} h\right)$ in the Extension Theorem, instead of a bigger one.

Now, we are ready to solve formally the non-homogeneous equation

$$
L \eta=\phi
$$

using the method of variation of parameters (see, for instance, [Gel96]). 
Lemma 3.2 The formal general solution of the non-homogeneous equation (3.11) has the form $\eta(t)=\eta_{\mathrm{h}}(t)+\eta_{\mathrm{p}}(t)$, where $\eta_{\mathrm{h}}(t)$ is the homogeneous solution (3.8), and a particular solution $\eta_{\mathrm{p}}(t)$ is

$$
\eta_{\mathrm{p}}(t)=\sum_{k \geq 1} \mathcal{N}_{k}(t) \phi(t-k h), \quad \mathcal{N}_{k}(t)=\eta_{2}(t) \eta_{1}(t-k h)-\eta_{2}(t-k h) \eta_{1}(t)
$$

Direct computations yield this lemma. The fact that the Wronskian of $\left\{\eta_{1}, \eta_{2}\right\}$ is identically one has been used. Otherwise, the term $\phi(t-k h)$ in the formula of the particular solution would be $\phi(t-k h) / W(t-k h)$.

In order to invert the linear operator $L$, we now study the convergence of the series in (3.12) for $\delta \in[h, \pi / 2]$. In the sequel, $K_{*}, K_{*}^{\prime}$ and $K_{*}^{\prime \prime}$ will denote positive constants independent of the parameters $k, h$ and $\varepsilon$, “*” being any subscript.

Lemma 3.3 Let $0<h \leq \delta \leq \pi / 2$. Then, the linear operator $L$ can be inverted on the complex space $\mathcal{X}_{\delta}$, and its inverse is analytic. Moreover, for $\phi \in \mathcal{X}_{\delta}$ and $t \in \mathcal{D}_{\delta}$

$$
L^{-1} \phi(t)=\sum_{k \geq 1} \mathcal{N}_{k}(t) \phi(t-k h), \quad\left|\mathcal{N}_{k}(t)\right| \leq K_{\mathcal{N}} \delta^{-2} h^{-1} \mathrm{e}^{k h}
$$

where $\mathcal{N}_{k}(t)$ is the function defined in (3.12).

Proof. We first notice that $\eta_{1}(t)$ is $\pi$ i-antiperiodic, but $\eta_{2}(t)$ is not $\pi$ i-antiperiodic, due to the term $(t-c) \tanh t / \cosh ^{2} t$. However, a straightforward computation shows that the function $\mathcal{N}_{k}(t)$ is $\pi \mathrm{i}$-periodic and does not depend on the constant $c$.

Second, on $\Im t \geq 0, \eta_{1}(t), \eta_{2}(t)$ have a singularity at $t_{\mathrm{p}}^{+}=\pi \mathrm{i} / 2$, whereas on $\Im t \leq 0$, the singularity is at $t_{\mathrm{p}}^{-}=-\pi \mathrm{i} / 2$. Because of this, we shall work separately on the sets $\mathcal{D}_{\delta}^{ \pm}=\mathcal{D}_{\delta} \cap\{ \pm \Im z \geq 0\}$ to get the same bound on the whole set $\mathcal{D}_{\delta}$.

We choose the value

$$
c=t_{\mathrm{p}}^{ \pm}= \pm \pi \mathrm{i} / 2
$$

for the free constant $c$ that appears in the expression (3.10) of $\eta_{2}(t)$. Then, there exists a constant $K_{\eta}$ such that

$$
\left|\eta_{1}(t)\right| \leq K_{\eta} \delta^{-2} h \mathrm{e}^{\Re t}, \quad\left|\eta_{2}(t)\right| \leq K_{\eta} h^{-2} \mathrm{e}^{-\Re t}, \quad \forall t \in \mathcal{D}_{\delta}^{ \pm} .
$$

The first bound is direct, but the second one requires a detailed verification. We split the function $\eta_{2}(t)$ as the sum $\eta_{2}(t)=\widehat{\eta}_{2}(t)+\widetilde{\eta}_{2}(t)$, where

$$
\widehat{\eta}_{2}(t)=\frac{2-\sinh ^{2} t-3\left(t-t_{\mathrm{p}}^{ \pm}\right) \tanh t}{2 \gamma^{2} \cosh t}, \quad \tilde{\eta}_{2}(t)=\frac{\widetilde{A}_{1}+\widetilde{A}_{2} \sinh ^{2} t+\widetilde{A}_{3}\left(t-t_{\mathrm{p}}^{ \pm}\right) \tanh t}{\cosh t}
$$

with

$$
\widetilde{A}_{1}=\frac{A_{1}-1}{\gamma^{2}}=\frac{\mu^{2}-1}{\gamma^{2}}=1, \quad \widetilde{A}_{2}=\frac{A_{2}+1 / 2}{\gamma^{2}}=0, \quad \widetilde{A}_{3}=\frac{A_{3}+3 / 2}{\gamma^{2}}=\frac{3(h-\gamma \mu)}{2 h \gamma^{2}} .
$$


On the one hand, since $\widetilde{A}_{1}, \widetilde{A}_{3}=\mathrm{O}(1), \widetilde{A}_{2}=0$, and $\delta \geq h$, there exists a constant $K_{\eta}^{\prime}$ such that

$$
\left|\widetilde{\eta}_{2}(t)\right| \leq K_{\eta}^{\prime} \delta^{-1} \leq K_{\eta}^{\prime} h^{-1} \leq \frac{\pi}{2} K_{\eta}^{\prime} \mathrm{e}^{T} h^{-2} \mathrm{e}^{-\Re t}, \quad \forall t \in \mathcal{D}_{\delta}^{ \pm} .
$$

On the other hand, the numerator of $\widehat{\eta}_{2}(t)$ has a zero at $t=t_{\mathrm{p}}^{ \pm}= \pm \pi \mathrm{i} / 2$, which compensates the zero of its denominator $\cosh t$, so $\widehat{\eta}_{2}(t)$ is analytic on $t=t_{\mathrm{p}}^{ \pm}$and there exists a constant $K_{\eta}^{\prime \prime}$ such that

$$
\left|\widehat{\eta}_{2}(t)\right| \leq K_{\eta}^{\prime \prime} h^{-2} \mathrm{e}^{-\Re t}, \quad \forall t \in \mathcal{D}_{\delta}^{ \pm}
$$

Thus, we can take $K_{\eta}=K_{\eta}^{\prime} \mathrm{e}^{T}+K_{\eta}^{\prime \prime}$ in the bound of $\eta_{2}$. This ends the proof of bound (3.14). Now, we are ready to bound $\mathcal{N}_{k}(t)$. If $K_{\mathcal{N}}=2\left(K_{\eta}\right)^{2}$, using (3.14), we deduce the bound in $(3.13)$ on $\mathcal{D}_{\delta}^{ \pm}$, and hence, on $\mathcal{D}_{\delta}$ :

$$
\begin{aligned}
\left|\mathcal{N}_{k}(t)\right| & \leq\left|\eta_{1}(t-k h)\right|\left|\eta_{2}(t)\right|+\left|\eta_{1}(t)\right|\left|\eta_{2}(t-k h)\right| \leq\left(K_{\eta}\right)^{2} \delta^{-2} h^{-1}\left(\mathrm{e}^{-k h}+\mathrm{e}^{k h}\right) \\
& \leq K_{\mathcal{N}} \delta^{-2} h^{-1} \mathrm{e}^{k h} .
\end{aligned}
$$

Let $\phi \in \mathcal{X}_{\delta}$. We know that the formal general solution of the equation $L \eta=\phi$ is $\eta=\eta_{\mathrm{h}}+\eta_{\mathrm{p}}$, where the functions $\eta_{\mathrm{h}}$ and $\eta_{\mathrm{p}}$ are given in (3.8) and (3.12), respectively. The particular solution $\eta_{\mathrm{p}}$ is analytic. Actually $\eta_{\mathrm{p}} \in \mathcal{X}_{\delta}$, since $\mathcal{N}_{k}(t)$ is $\pi$ i-periodic, and

$$
\left\|\eta_{\mathrm{p}}\right\|_{\delta} \leq K_{\mathcal{N}} \delta^{-2} h^{-1} \frac{\mathrm{e}^{-2 h}}{1-\mathrm{e}^{-2 h}}\|\phi\|_{\delta}
$$

as the following computation shows

$$
\mathrm{e}^{-3 \Re t}\left|\eta_{\mathrm{p}}(t)\right| \leq \mathrm{e}^{-3 \Re t} \sum_{k \geq 1}\left|\mathcal{N}_{k}(t)\right||\phi(t-k h)| \leq K_{\mathcal{N}}\|\phi\|_{\delta} \delta^{-2} h^{-1} \sum_{k \geq 1} \mathrm{e}^{-2 k h}, \quad \forall t \in \mathcal{D}_{\delta} .
$$

(We have used that $|\phi(t)| \leq \mathrm{e}^{3 \Re t}\|\phi\|_{\delta}$, on $\mathcal{D}_{\delta}$.)

Since $\eta_{1}, \eta_{2}$, and $\eta_{\mathrm{p}}$ are analytic on $\mathcal{D}_{\delta}$, it turns out that the formal solutions $\eta_{\mathrm{h}}+\eta_{\mathrm{p}}$ of $L \eta=\phi$ are analytic on $\mathcal{D}_{\delta}$, provided that the $h$-periodic functions $\alpha_{1}(t)$ and $\alpha_{2}(t)$ in (3.8) are analytic, too. Furthermore, $\left|\eta_{1}(t)\right| \sim 2 \gamma \mathrm{e}^{-\Re t}$ and $\left|\eta_{2}(t)\right| \sim \frac{1}{4} \gamma^{-2} \mathrm{e}^{\Re t}$, when $\Re t \rightarrow-\infty$ and $\Im t=0$. Thus, $\left\|\eta_{\mathrm{h}}\right\|_{\delta}<\infty$ if and only if $\alpha_{1}(t), \alpha_{2}(t) \equiv 0$, that is, $\eta_{\mathrm{h}}(t) \equiv 0$. This proves that $\eta=\eta_{\mathrm{p}}$ is the unique solution in $\mathcal{X}_{\delta}$ of the equation $L \eta=\phi$, so $L$ is invertible in $\mathcal{X}_{\delta}$ and $L^{-1}$ is given by (3.12).

Moreover $\left\|L^{-1}\right\|_{\delta} \leq K_{\mathcal{N}} \delta^{-2} h^{-1} \mathrm{e}^{-2 h} /\left(1-\mathrm{e}^{-2 h}\right)$, see (3.15). Thus $L^{-1}$ is a linear continuous operator between complex Banach spaces and, therefore, analytic.

\subsection{The nonlinear equation}

Once we know how to solve the linear equation (3.11), we are ready to study the nonlinear one $L \eta=\mathcal{T} \eta$. Since our goal is to prove proposition 3.1, we shall work in the spaces $\mathcal{X}_{h}$, and we shall omit the subscript $h$ in the norm of $\mathcal{X}_{h}$, to avoid a cumbersome notation. 
Introducing $\mathcal{B}_{R}=\left\{\eta \in \mathcal{X}_{h}:\|\eta\|<R\right\}$, we claim that the operators

$$
\mathcal{B}_{R} \ni \eta(t) \stackrel{\mathcal{T}}{\longmapsto} \Phi(t, \eta(t)) \in \mathcal{X}_{h}, \quad \mathcal{B}_{R} \ni \eta(t) \stackrel{\mathcal{F}}{\longmapsto} L^{-1} \Phi(t, \eta(t))=L^{-1} \mathcal{T} \eta(t) \in \mathcal{X}_{h}
$$

are well-defined and analytic for $R=\mathrm{e}^{-3 T} / 4>0$ (see lemma 3.5). Thus, $\eta \in \mathcal{B}_{R} \subset \mathcal{X}_{h}$ is a solution of the fixed point equation $\eta=\mathcal{F} \eta=L^{-1} \mathcal{T} \eta$, if and only if $L \eta=\mathcal{T} \eta$. Consequently, to prove proposition 3.1 it suffices to see that, under the hypotheses there stated, the operator $\mathcal{F}$ has a fixed point $\eta^{*} \in \mathcal{X}_{h}$ such that $\left\|\eta^{*}\right\|=\mathrm{O}\left(\varepsilon h^{3}\right)$.

In order to solve the fixed point equation, we shall use a contraction argument. Since $\mathcal{F}$ is an analytic operator on a complex Banach space the usual contraction lemma admits a stronger formulation. In practice this stronger version does not give anything new with regard to the usual one, but it exempts us of the tedious computation which show that the operator we consider is a contraction. We learned this trick from [Ang93]. The adaptation of this idea to our setting is the following lemma.

Lemma 3.4 (Analytic Contraction Lemma) Let $\mathcal{X}$ be a complex Banach space, and let $\mathcal{F}: \mathcal{B}_{R} \rightarrow \mathcal{X}$ be an analytic map, where $\mathcal{B}_{R}=\{\eta \in \mathcal{X}:\|\eta\|<R\}$. Assume that

$$
\|\mathcal{F} \eta\| \leq a+b\|\eta\|+c\|\eta\|^{2}+d\|\eta\|^{3}, \quad \forall \eta \in \mathcal{B}_{R}
$$

with

$$
4 a<R, \quad 4 b+16 a c+64 a^{2} d<1 .
$$

Then $\mathcal{F}$ has a unique fixed point $\eta^{*} \in \mathcal{X}$ such that $\left\|\eta^{*}\right\|<2 a$.

Proof. Suppose first that $\mathcal{F}\left(\mathcal{B}_{r}\right) \subset \mathcal{B}_{\theta r}$, for some $r \in(0, R)$ and $\theta<1 / 2$. Let $\eta \in \mathcal{B}_{\theta r}$ and $\rho=r-\|\eta\|>0$. The Cauchy's inequality to estimate the derivative of $\mathcal{F}$ in $\eta$ gives

$$
\begin{aligned}
\|\mathrm{d} \mathcal{F}(\eta)\| & \leq \rho^{-1} \sup \{\|\mathcal{F} \phi\|:\|\phi-\eta\|<\rho\} \leq(r-\|\eta\|)^{-1} \sup \left\{\|\mathcal{F} \phi\|: \phi \in \mathcal{B}_{r}\right\} \\
& \leq \frac{\theta r}{r-\theta r}=\frac{\theta}{1-\theta}<1,
\end{aligned}
$$

where we have used that $\theta<1 / 2$. Thus, the restriction of $\mathcal{F}$ to the ball $\mathcal{B}_{\theta r}$ is a contraction, and hence $\mathcal{F}$ has a unique fixed point $\eta^{*} \in \mathcal{B}_{\theta r} \subset \mathcal{B}_{r / 2}$.

To finish, it suffices to note that hypothesis (3.17) implies $\mathcal{F}\left(\mathcal{B}_{r}\right) \subset \mathcal{B}_{\theta r}$, with $r=$ $4 a<R$ and $\theta=\left(1+4 b+16 a c+64 a^{2} d\right) / 4<1 / 2$.

We will see in lemma 3.5 that the nonlinear operator $\mathcal{F}$ satisfies bound (3.16) with

$$
a=\mathrm{O}\left(\varepsilon h^{-3}\right), \quad b=\mathrm{O}\left(\varepsilon h^{-3}\right), \quad c=\mathrm{O}\left(h^{-3} \ln h\right), \quad d=\mathrm{O}\left(h^{-4}\right),
$$

when $h$ and $|\varepsilon|$ are small. Therefore,

$$
4 a=\mathrm{O}\left(\varepsilon h^{-3}\right), \quad 4 b+16 a c+64 a^{2} d=\mathrm{O}\left(\varepsilon h^{-6} \ln h, \varepsilon^{2} h^{-10}\right),
$$

and hypotheses (3.17) of the Analytic Contraction Lemma hold for $h$ and $\left|\varepsilon h^{-6} \ln h\right|$ small enough. This proves that, under the hypotheses of the proposition 3.1, the operator $\mathcal{F}$ has a fixed point $\eta^{*} \in \mathcal{X}_{h}$ such that $\left\|\eta^{*}\right\|<2 a=\mathrm{O}\left(\varepsilon h^{-3}\right)$.

Therefore, proposition 3.1, and the Extension Theorem, are proved. 
Remark 3.2 The bound $a=\mathrm{O}\left(\varepsilon h^{-3}\right)$ is the direct responsible of bound (3.6), which in its turn, together with the bound $c=\mathrm{O}\left(h^{-3} \ln h\right)$, is the cause of the hypothesis $h$ small and $\varepsilon=\mathrm{o}\left(h^{6} \ln ^{-1} h\right)$ in proposition 3.1. Besides, the value of the operator $\mathcal{F}$ at the point $0 \in \mathcal{X}_{h}$ can be easily computed:

$$
\mathcal{F} 0: t \mapsto L^{-1} \Phi(t, 0)=\varepsilon L^{-1} V^{\prime}\left(\xi^{0}(t)\right)=\varepsilon \sum_{k \geq 1} \mathcal{N}_{k}(t) V^{\prime}\left(\xi^{0}(t-k h)\right)
$$

and numerical experiments show that $\|\mathcal{F} 0\| \simeq \varepsilon h^{-3}$, when $h$ and $|\varepsilon|$ are small. (The experiments have been performed with $V^{\prime}(y)=y^{3}$, that is, the simplest perturbation satisfying our hypotheses that we can take.) Thus, the bound on $a$ is optimal because $\|\mathcal{F} 0\| \leq a$. This fact leads us to believe that bound (3.6) is optimal. This belief also relies on additional numerical experiments, which also show that bound (3.6) holds under the weaker hypothesis $h$ small and $\varepsilon=\mathrm{o}\left(h^{3}\right)$.

It only remains to prove the properties of the operators $\mathcal{T}$ and $\mathcal{F}$ we have used before, and this is the aim of the following lemma.

Lemma 3.5 If $h_{0}$ is small enough, $h \in\left(0, h_{0}\right)$, and $t \in \mathcal{D}_{h}$, the following bounds hold:

(i) $\left|\xi^{0}(t) \pm \mathrm{i}\right| \geq 1 / 2$.

(ii) $\left|\mathcal{N}_{k}(t)\right| \leq K_{\mathcal{N}} h^{-3} \mathrm{e}^{k h}$, where $\mathcal{N}_{k}(t)$ is the function defined in (3.12).

(iii) Let $\Phi(t, \eta)$ be the function introduced in (3.3)-(3.4). Then,

$$
|\Phi(t, \eta)| \leq K_{\Phi}\left\{\sum_{q=2}^{3}|\eta|^{q}\left|\xi^{0}(t)\right|^{3-q}+|\varepsilon| \sum_{q=0}^{3}|\eta|^{q}\left|\xi^{0}(t)\right|^{3-q}\right\}, \quad \forall \eta \in B_{1 / 4} .
$$

Moreover, setting $\mathcal{B}_{R}=\left\{\eta \in \mathcal{X}_{h}:\|\eta\|<R\right\}$ with $R=\mathrm{e}^{-3 T} / 4$, the nonlinear operator

$$
\mathcal{B}_{R} \ni \eta(t) \stackrel{\mathcal{T}}{\longrightarrow} \Phi(t, \eta(t)) \in \mathcal{X}_{h}
$$

is well-defined and analytic.

(iv) Let $\mathcal{M}_{q}(t)=\sum_{k \geq 1} \exp [3(q-1) \Re t-(3 q-1) k h]\left|\xi^{0}(t-k h)\right|^{3-q}$, for $q=0,1,2,3$. Then,

$$
\mathcal{M}_{q}(t) \leq \begin{cases}K_{\mathcal{M}} \mathrm{e}^{2 q \Re t} & \text { if } q=0,1 \\ K_{\mathcal{M}}|\ln h| \mathrm{e}^{4 \Re t} & \text { if } q=2 \\ K_{\mathcal{M}} h^{-1} \mathrm{e}^{6 \Re t} & \text { if } q=3\end{cases}
$$

(v) For $R=\mathrm{e}^{-3 T} / 4$, the nonlinear operator

$$
\mathcal{B}_{R} \ni \eta(t) \stackrel{\mathcal{F}}{\longmapsto} L^{-1} \Phi(t, \eta(t)) \in \mathcal{X}_{h}
$$

is well-defined and analytic on $\mathcal{B}_{R}$. Furthermore, for all $\eta \in \mathcal{B}_{R}$,

$$
\|\mathcal{F} \eta\| \leq K_{\mathcal{F}} h^{-3}\left\{|\varepsilon|(1+\|\eta\|)+(1+|\varepsilon|)|\ln h|\|\eta\|^{2}+(1+|\varepsilon|) h^{-1}\|\eta\|^{3}\right\} .
$$


Proof. The value of $h_{0}$ can change along the proof, becoming smaller and smaller, without explicit mention. Thus, we will use freely bounds like $h<1, \ln h<-1, \gamma<2 h$, $\mu<2$, or any other one that holds near the limit $h=0$.

(i) We will obtain the bound separately on the sets $\mathcal{J}$ and $\mathcal{K}_{h}$. (Recall that $\mathcal{D}_{h}=$ $\mathcal{J} \cup \mathcal{K}_{h}$, see equation (3.5).) The bound on $\mathcal{J}$ is trivial, since $\xi^{0}$ tends uniformly to zero on $\mathcal{J}$, when $h \rightarrow 0$.

We note that $\xi^{0}( \pm h+\pi \mathrm{i} / 2)=\mathrm{i}$ and $\xi^{0}( \pm h-\pi \mathrm{i} / 2)=-\mathrm{i}$, so the bound on $\mathcal{K}_{h}$ is more difficult. Let $r=\Re t, s=\Im t$. Elementary computations give

$$
\left|\xi^{0}(t)+\mathrm{i}\right|^{2}=1+\frac{\gamma(\gamma-2 \sinh r \sin s)}{\sinh ^{2} r+\cos ^{2} s} \geq 1+\frac{\gamma(\gamma-2 \sinh |r|)}{\sinh ^{2} r+\cos ^{2} s} .
$$

If $\gamma-2 \sinh |r| \geq 0$, then $\left|\xi^{0}(t)+\mathrm{i}\right|^{2} \geq 1>1 / 4$. Otherwise, using the inequality $\cos ^{2} s=\cos ^{2}(\Im t) \geq \sin ^{2} h$, for $t \in \mathcal{K}_{h}$, we obtain

$$
\left|\xi^{0}(t)+\mathrm{i}\right|^{2} \geq 1+\frac{\gamma(\gamma-2 \sinh |r|)}{\sinh ^{2} r+\sin ^{2} h}=1+\frac{1-2 \alpha}{\alpha^{2}+c(h)^{2}}=: g_{h}(\alpha),
$$

where $\alpha=\gamma^{-1} \sinh |r|$, and $c(h)=\gamma^{-1} \sin h$. In particular, $\left|\xi^{0}(t)+\mathrm{i}\right|^{2} \geq \min _{\mathbb{R}} g_{h}$, for $t \in \mathcal{K}_{h}$. Let $g_{0}(\alpha)=1+(1-2 \alpha) /\left(\alpha^{2}+1\right)$. The function $g_{h}$ converges uniformly to $g_{0}$ on the real line, when $h \rightarrow 0$, and $\min _{\mathbb{R}} g_{0}=g_{0}((1+\sqrt{5}) / 2)=0.382 \ldots$, so $\min _{\mathbb{R}} g_{h}>1 / 4$, for $h$ small enough. This finishes the proof of the bound $\left|\xi^{0}(t)+\mathrm{i}\right| \geq 1 / 2$, for $t \in \mathcal{K}_{h}$.

The bound on $\left|\xi^{0}(t)-\mathrm{i}\right|$ is similar.

(ii) It is a direct consequence of (3.13) for $\delta=h$.

(iii) Let us recall that $\Phi=\Phi^{0}+\varepsilon \Phi^{1}$. In order to bound the function $\Phi^{0}$, we write $\Phi^{0}=\widehat{\Phi}^{0}+\widetilde{\Phi}^{0}$, where

$$
\widehat{\Phi}^{0}(t, \eta)=2 \mu \frac{\widehat{P}\left(\xi^{0}(t)\right)}{Q\left(\xi^{0}(t), \eta\right)} \xi^{0}(t) \eta^{2}, \quad \tilde{\Phi}^{0}(t, \eta)=2 \mu \frac{\widetilde{P}\left(\xi^{0}(t)\right)}{Q\left(\xi^{0}(t), \eta\right)} \eta^{3},
$$

with

$$
\widehat{P}(\xi)=\xi^{2}-3, \quad \tilde{P}(\xi)=\xi^{2}-1, \quad Q(\xi, \eta)=\left(1+\xi^{2}\right)^{2}\left[1+(\xi+\eta)^{2}\right] .
$$

Now, we can control the size of the denominator $Q\left(\xi^{0}(t), \eta\right)$ using (i). In particular,

$$
\left|Q\left(\xi^{0}(t), \eta\right)\right| \geq 2^{-8}, \quad \forall \eta \in B_{1 / 4}, \forall t \in \mathcal{D}_{h} .
$$

Moreover, there exists a constant $K_{P}$ such that

$$
\left|\widehat{P}\left(\xi^{0}(t)\right)\right|,\left|\widetilde{P}\left(\xi^{0}(t)\right)\right| \leq K_{P}, \quad \forall t \in \mathcal{D}_{h},
$$

since $\xi^{0}(t)=\gamma$ sech $t$ is uniformly bounded on $\mathcal{D}_{h}$, and $\widehat{P}(\xi), \widetilde{P}(\xi)$ are polynomial functions. Thus, using that $\mu<2$,

$$
\left|\Phi^{0}(t, \eta)\right| \leq 2^{10} K_{P} \sum_{q=2}^{3}|\eta|^{q}\left|\xi^{0}(t)\right|^{3-q}, \quad \forall \eta \in B_{1 / 4}, \forall t \in \mathcal{D}_{h} .
$$


We now proceed to bound $\Phi^{1}(t, \eta)=V^{\prime}\left(\xi^{0}(t)+\eta\right)$. As $y=0$ is a triple zero of $V^{\prime}(y)$, $V^{\prime}(y) / y^{3}$ is an entire function. In particular, there exists a constant $K_{V}$ such that

$$
\left|\Phi^{1}(t, \eta)\right| \leq K_{V}\left|\xi^{0}(t)+\eta\right|^{3} \leq 3 K_{V} \sum_{q=0}^{3}|\eta|^{q}\left|\xi^{0}(t)\right|^{3-q}, \quad \forall \eta \in B_{1 / 4}, \forall t \in \mathcal{D}_{h} .
$$

(We have used again the fact that $\xi^{0}(t)$ is uniformly bounded on $\mathcal{D}_{h}$.) The bounds $(3.18$ ) and (3.19) imply the first part of (iii); it suffices to take $K_{\Phi}=\max \left\{2^{10} K_{P}, 3 K_{V}\right\}$.

In order to get the properties of the operator $\mathcal{T}$, let us fix a function $\eta \in \mathcal{B}_{R}$. Then,

$$
|\eta(t)| \leq \mathrm{e}^{3 \Re t}\|\eta\|<\mathrm{e}^{3 T} R=1 / 4, \quad \forall t \in \mathcal{D}_{h} .
$$

Therefore, the function $t \mapsto \mathcal{T} \eta(t):=\Phi(t, \eta(t))$ is analytic on $\mathcal{D}_{h}$, because $\Phi(t, \eta)$ is analytic on $(t, \eta) \in \mathcal{D}_{h} \times B_{1 / 4}$. Moreover, an easy computation shows that $\mathcal{T} \eta \in \mathcal{X}_{h}$, so the operator $\mathcal{T}$ is well-defined and analytic.

(iv) There exists a constant $K_{\mathrm{e}}$ such that $\sum_{k \geq 1} \mathrm{e}^{-n h k} \leq\left(1-\mathrm{e}^{-n h}\right)^{-1} \leq K_{\mathrm{e}} h^{-1}$, for $n=2,4,6,8$. Set

$$
\tau=\left\{\begin{array}{ll}
t-\pi \mathrm{i} / 2 & \text { if } \Im t \geq 0 \\
t+\pi \mathrm{i} / 2 & \text { otherwise }
\end{array} .\right.
$$

Then, there exists a constant $K_{\xi}$ such that

$$
\left|\xi^{0}(t)\right|^{3-q} \leq\left\{\begin{array}{ll}
K_{\xi} h^{3-q} \mathrm{e}^{(3-q) \Re t} & \text { on } \mathcal{J} \\
K_{\xi} h^{3-q}|\tau|^{q-3} & \text { on } \mathcal{K}_{h}
\end{array} \quad \forall q=0,1,2 .\right.
$$

The case $q=3$ is easy:

$$
\mathcal{M}_{3}(t)=\mathrm{e}^{6 \Re t} \sum_{k \geq 1} \mathrm{e}^{-8 k h} \leq K_{\mathrm{e}} h^{-1} \mathrm{e}^{6 \Re t}, \quad \forall t \in \mathcal{D}_{h}
$$

Now, we consider the case $q \neq 3$. We work again separately on the sets $\mathcal{J}, \mathcal{K}_{h}$. On $\mathcal{J}$ the bound is

$$
\mathcal{M}_{q}(t) \leq K_{\xi} h^{3-q} \mathrm{e}^{2 q \Re t} \sum_{k \geq 1} \mathrm{e}^{-(2+2 q) k h} \leq K_{\xi} K_{\mathrm{e}} h^{2-q} \mathrm{e}^{2 q \Re t}
$$

and setting $K_{\mathcal{M}}^{\prime}=K_{\xi} K_{\mathrm{e}}$

$$
\mathcal{M}_{q}(t) \leq\left\{\begin{array}{ll}
K_{\mathcal{M}}^{\prime} \mathrm{e}^{2 q \Re t} & \text { if } q=0,1 \\
K_{\mathcal{M}}^{\prime}|\ln h| \mathrm{e}^{4 \Re t} & \text { if } q=2
\end{array}, \quad \forall t \in \mathcal{J}\right.
$$

To end, let us assume that there exists a constant $K_{\mathcal{M}}^{\prime \prime}$ verifying

$$
\mathcal{M}_{q}(t) \leq\left\{\begin{array}{ll}
K_{\mathcal{M}}^{\prime \prime} & \text { if } q=0,1 \\
K_{\mathcal{M}}^{\prime \prime}|\ln h| & \text { if } q=2
\end{array}, \quad \forall t \in \mathcal{K}_{h}\right.
$$

Then, setting $K_{\mathcal{M}}=\max \left\{K_{\mathcal{M}}^{\prime}, \mathrm{e}^{4 T} K_{\mathcal{M}}^{\prime \prime}, K_{\mathrm{e}}\right\}$, (iv) follows from equations $(3.20)-(3.22)$. 
We now proceed to prove bound (3.22). Given $t \in \mathcal{K}_{h}$, let $\ell=\ell(t)$ be the smallest positive integer such that $t-\ell h \in \mathcal{J}$, and split the series $\mathcal{M}_{q}(t)$ as

$$
\begin{aligned}
\mathcal{M}_{q}(t) & =\mathcal{M}_{q}(t-\ell h)+\sum_{k=1}^{\ell-1} \exp [3(q-1) \Re t-(3 q-1) k h]\left|\xi^{0}(t-k h)\right|^{3-q} \\
& \leq \max _{t \in \mathcal{J}} \mathcal{M}_{q}(t)+K_{\xi} \mathrm{e}^{3 T} h^{3-q} \sum_{k=1}^{\ell-1}|\tau-k h|^{q-3}, \quad t \in \mathcal{K}_{h} .
\end{aligned}
$$

We remember that $|\Im \tau| \geq h$, and $-T / h+\ell-1 \leq \Re \tau / h \leq T / h$. Using the bound $\sum_{k=1}^{\ell-1} f(k) \leq \max _{\mathbb{R}} f+\int_{1}^{\ell} f$, valid for any positive continuous function $f$ such that it is increasing on $(-\infty, r)$ and decreasing on $(r,+\infty)$ for some $r \in \mathbb{R}$, we get

$$
\begin{aligned}
h^{3-q} \sum_{k=1}^{\ell-1}|\tau-k h|^{q-3} & \leq h^{3-q} \sum_{k=1}^{\ell-1}|\Re \tau-k h+h \mathrm{i}|^{q-3}=\sum_{k=1}^{\ell-1}|\Re \tau / h-k+\mathrm{i}|^{q-3} \\
& \leq 1+\int_{1}^{\ell}|\Re \tau / h-u+\mathrm{i}|^{q-3} \mathrm{~d} u=1+\int_{\Re \tau / h-\ell}^{\Re \tau / h-1}|v+\mathrm{i}|^{q-3} \mathrm{~d} v \\
& \leq 1+\int_{-T / h-1}^{T / h-1} \frac{\mathrm{d} v}{\left(1+v^{2}\right)^{(3-q) / 2}}=\left\{\begin{array}{ll}
\mathrm{O}(1) & \text { if } q=0,1 \\
\mathrm{O}(\ln h) & \text { if } q=2
\end{array} .\right.
\end{aligned}
$$

Now, bound (3.22) follows from these last two estimates and bound (3.21).

(v) The fact that $\mathcal{F}$ is well-defined and analytic on $\mathcal{B}_{R}$ is obvious, because it is the composition of two operators, $\mathcal{T}: \mathcal{B}_{R} \rightarrow \mathcal{X}_{h}$ and $L^{-1}: \mathcal{X}_{h} \rightarrow \mathcal{X}_{h}$, well-defined and analytic. Consequently, we fix our attention on the bound.

Let $\eta \in \mathcal{B}_{R}$, and set $K_{\mathcal{F}}=K_{\mathcal{N}} K_{\Phi} K_{\mathcal{M}}$. Then, using the bounds from (ii)-(iv), we get

$$
\begin{aligned}
\mathrm{e}^{-3 \Re t}|\mathcal{F} \eta(t)| \leq & \mathrm{e}^{-3 \Re t} \sum_{k \geq 1}\left|\mathcal{N}_{k}(t)\right||\Phi(t-k h, \eta(t-k h))| \\
\leq & K_{\mathcal{N}} K_{\Phi} h^{-3} \sum_{k \geq 1} \mathrm{e}^{-3 \Re t+k h}\left\{\sum_{q=2}^{3}|\eta(t-k h)|^{q}\left|\xi^{0}(t-k h)\right|^{3-q}\right. \\
& \left.+|\varepsilon| \sum_{q=0}^{3}|\eta(t-k h)|^{q}\left|\xi^{0}(t-k h)\right|^{3-q}\right\} \\
\leq & K_{\mathcal{N}} K_{\Phi} h^{-3}\left\{\sum_{q=2}^{3}|| \eta\left\|^{q} \mathcal{M}_{q}(t)+|\varepsilon| \sum_{q=0}^{3}\right\| \eta \|^{q} \mathcal{M}_{q}(t)\right\} \\
\leq & K_{\mathcal{F}} h^{-3}\left\{|\varepsilon|(1+\|\eta\|)+(1+|\varepsilon|)|\ln h|\|\eta\|^{2}+(1+|\varepsilon|) h^{-1}|| \eta \|^{3}\right\}
\end{aligned}
$$

for all $t \in \mathcal{D}_{h}$.

Remark 3.3 Let $\delta \in(0, \pi / 2]$ be fixed, i.e., independent of $h$. Then, the analysis on $\mathcal{D}_{\delta}$ is simpler than the one on $\mathcal{D}_{h}$, since we are far from the singularities. The bounds (i) 
and (iii) of the previous lemma are the same, whereas the bounds (ii) and (iv) can be improved in the following way:

$$
\left|\mathcal{N}_{k}(t)\right| \leq K_{\mathcal{N}} h^{-1} \mathrm{e}^{k h}, \quad \mathcal{M}_{q}(t) \leq K_{\mathcal{M}} h^{2-q} \mathrm{e}^{2 q \Re t}, \quad q=0,1,2,3
$$

for all $t \in \mathcal{D}_{\delta}$. Thus, repeating the argument of $(\mathrm{v})$, the operator $\mathcal{F}$ is well-defined and analytic on $\mathcal{B}_{R}=\left\{\eta \in \mathcal{X}_{\delta}:\|\eta\|_{\delta}<R\right\}$, and verifies bound (3.16) as before, but now with

$$
a=\mathrm{O}(\varepsilon h), \quad b=\mathrm{O}(\varepsilon), \quad c=\mathrm{O}\left(h^{-1}\right), \quad d=\mathrm{O}\left(h^{-2}\right),
$$

when $h$ and $|\varepsilon|$ are small. Therefore,

$$
4 a=\mathrm{O}(\varepsilon h), \quad 4 b+16 a c+64 a^{2} d=\mathrm{O}(\varepsilon),
$$

and the hypotheses (3.17) of the Analytic Contraction Lemma are verified for $h$ and $|\varepsilon|$ small enough. This proves that, under these hypotheses, the operator $\mathcal{F}$ has a fixed point $\eta^{*} \in \mathcal{X}_{\delta}$ such that $\left\|\eta^{*}\right\|_{\delta}<2 a=\mathrm{O}(\varepsilon h)$. Hence, in the same way that we have proved the Extension Theorem, bound (2.15) follows. We omit the details, since they do not require any new idea.

\section{Proof of Proposition 2.2}

(a) Let $B$ the matrix such that $H_{0}(z)=\frac{1}{2} z^{\top} B z+\mathrm{O}\left(h^{-1} z^{4}\right)$. A direct computation shows that $B C=\mathrm{O}(h)$, and hence $\mathrm{d} H^{0}(z)=\mathrm{O}(h)$, for $z \in U=C \Phi(\mathcal{U})$. Assume that $T / 2 \leq \Re t \leq T$ and $|\Im t| \leq \pi / 2-h$. Then, bounds (2.14) and (2.6) hold and $z^{\mathrm{u}, \mathrm{s}}(t) \in U$. Applying Taylor's Theorem to $H^{0}\left(z^{\mathrm{u}, \mathrm{s}}(t)\right)=H^{0}\left(z^{\mathrm{u}, \mathrm{s}}(t)\right)-H^{0}\left(z^{0}(t)\right)$ we get

$$
H^{0}\left(z^{\mathrm{u}}(t)\right)=\mathrm{O}\left(\varepsilon h^{-2}\right), \quad H^{0}\left(z^{\mathrm{s}}(t)\right)=\mathrm{O}\left(\varepsilon h^{2}\right) .
$$

These estimates together with equations (2.11)-(2.13) imply

$$
\begin{aligned}
E^{\mathrm{u}}(t)=E\left(z^{\mathrm{u}}(t)\right)-E\left(z^{\mathrm{s}}(t)\right) & =E^{0}\left(z^{\mathrm{u}}(t)\right)-E^{0}\left(z^{\mathrm{s}}(t)\right)+\mathrm{O}\left(\varepsilon h^{3}\right) \\
& =\Delta(t)+\mathrm{O}\left(\varepsilon h^{3}, \varepsilon^{2} h^{-4}\right)
\end{aligned}
$$

where

$$
\begin{aligned}
\Delta(t) & =H^{0}\left(z^{\mathrm{u}}(t)\right)-H^{0}\left(z^{\mathrm{s}}(t)\right) \\
& =H^{0}\left(z^{\mathrm{u}}\left(t_{-m}\right)\right)-H^{0}\left(z^{\mathrm{s}}\left(t_{m}\right)\right)+\sum_{n=-m}^{m-1} H^{0}\left(z^{\alpha(n)}\left(t_{n+1}\right)\right)-H^{0}\left(z^{\alpha(n)}\left(t_{n}\right)\right)
\end{aligned}
$$

for all $m>0$, where $t_{n}=t+h n$, and $\alpha(n)$ stands for $\mathrm{u}$ if $n<0$, and for $\mathrm{s}$ if $n \geq 0$. Since $\lim _{m \rightarrow \infty} z^{\mathrm{u}}\left(t_{-m}\right)=\lim _{m \rightarrow \infty} z^{\mathrm{s}}\left(t_{m}\right)=O=(0,0)$, and $z^{\mathrm{u}, \mathrm{s}}\left(t_{n}\right)=F\left(z^{\mathrm{u}, \mathrm{s}}\left(t_{n-1}\right)\right)$, we obtain by passing to the limit

$$
\Delta(t)=\sum_{n \in \mathbb{Z}} H^{0}\left(z^{\alpha(n)}\left(t_{n+1}\right)\right)-H^{0}\left(z^{\alpha(n)}\left(t_{n}\right)\right)=\sum_{n \in \mathbb{Z}}\left(H^{0} \circ F-H^{0}\right)\left(z^{\alpha(n)}\left(t_{n}\right)\right) .
$$


Adding and subtracting $\varepsilon M(t), M(t)$ being the Melnikov sum (1.9), we get

$$
\Delta(t)=\varepsilon M(t)+\varepsilon Q(t), \quad Q(t)=\sum_{n \in \mathbb{Z}}\left\{R\left(z^{\alpha(n)}\left(t_{n}\right)\right)-R^{0}\left(z^{0}\left(t_{n}\right)\right)\right\},
$$

where $R=\varepsilon^{-1}\left(H^{0} \circ F-H^{0}\right)$, for $\varepsilon \neq 0$, and $R=R^{0}=\left(\mathrm{d} H^{0} \circ F^{0}\right) \cdot F^{1}$, for $\varepsilon=0$.

Set $Q=Q_{+}+Q_{0}+Q_{-}$, with

$$
\begin{aligned}
Q_{+}(t) & =\sum_{n=-\infty}^{-N}\left\{R\left(z^{\mathrm{u}}\left(t_{n}\right)\right)-R^{0}\left(z^{0}\left(t_{n}\right)\right)\right\} \\
Q_{0}(t) & =\sum_{n=1-N}^{-1}\left\{R\left(z^{\mathrm{u}}\left(t_{n}\right)\right)-R^{0}\left(z^{0}\left(t_{n}\right)\right)\right\}, \\
Q_{-}(t) & =\sum_{n=0}^{+\infty}\left\{R\left(z^{\mathrm{s}}\left(t_{n}\right)\right)-R^{0}\left(z^{0}\left(t_{n}\right)\right)\right\},
\end{aligned}
$$

$N \leq 2 T h^{-1}$ being the smallest natural such that $t_{-N} \in \mathcal{J}^{\text {u }}$, i.e., $\Re t-N h \leq-T / 2$.

First, we focus our attention on the term $Q_{0}$. In order to bound it, we must estimate the size of the differences $R\left(z^{\mathrm{u}}\left(t_{n}\right)\right)-R\left(z^{0}\left(t_{n}\right)\right)$, and this requires a better knowledge of the function $R$. Let $\Omega=\left\{z=(x, y) \in \mathbb{C}^{2}: y \neq \pm \mathrm{i}\right\}$. Using the equalities $H^{0} \circ F^{0}=H^{0}$, $F=F^{0}+\varepsilon F^{1}$, it turns out that $R=R^{0}+\varepsilon R^{1}, h R^{0}(z, h)$ and $h R^{1}(z, h, \varepsilon)$ being analytic for all $z \in \Omega$, and $h, \varepsilon \in \mathbb{C}$. (The denominator $2 \gamma=\mathrm{O}(h)$ in the formula (1.5) for the first integral $H^{0}$ forces us to put the factor $h$, whereas the denominator $1+y^{2}$ in the definition of the McMillan map $F^{0}(z)$ forces us to work on $z \in \Omega$.) Hence, given a compact set $\Omega^{0} \subset \Omega$, there exists a positive constant $K$ such that

$$
\left|R\left(z^{\mathrm{u}}\right)-R^{0}\left(z^{0}\right)\right| \leq\left|\varepsilon R^{1}\left(z^{\mathrm{u}}\right)\right|+\left|R^{0}\left(z^{\mathrm{u}}\right)-R^{0}\left(z^{0}\right)\right| \leq K h^{-1}\left[|\varepsilon|+\left|z^{\mathrm{u}}-z^{0}\right|\right],
$$

for all $z^{\mathrm{u}}, z^{0} \in \Omega^{0}$.

Let $\Omega^{0}$ be the compact subset of $\Omega$ given by $\left\{z=(x, y) \in \mathbb{C}^{2}:|z| \leq 2,|y \pm i| \geq 1 / 2\right\}$. Item (i) of Lemma 3.5 implies that $z^{0}\left(\mathcal{K}_{h}\right) \subset \Omega^{0}$, for small enough values of $h$. On the other hand, equation (2.14) provides the bound $z^{\mathrm{u}}\left(t_{n}\right)-z^{0}\left(t_{n}\right)=\mathrm{O}\left(\varepsilon h^{-3}\right)$, since $t_{n} \in \mathcal{K}_{h}$, for $1-N \leq n \leq-1$. Therefore, $z^{\mathrm{u}}\left(t_{n}\right), z^{0}\left(t_{n}\right) \in \Omega^{0}$, for $1-N \leq n \leq-1$. Then, equation (4.3) provides the estimate $R\left(z^{\mathrm{u}}\left(t_{n}\right)\right)-R^{0}\left(z^{0}\left(t_{n}\right)\right)=\mathrm{O}\left(\varepsilon h^{-4}\right)$, and $Q_{0}=\mathrm{O}\left(\varepsilon h^{-5}\right)$, because there are $N-1=\mathrm{O}\left(h^{-1}\right)$ terms in the sum, each one of order $\mathrm{O}\left(\varepsilon h^{-4}\right)$.

The terms $Q_{ \pm}$can be estimated along the same lines. Of course, their bounds are smaller than the one for $Q_{0}$, because one uses the bounds (2.5)-(2.6), instead of the bigger one (2.14). The result is $Q_{ \pm}=\mathrm{O}\left(\varepsilon h^{-1}\right)$.

Collecting the bounds for $Q_{0}$ and $Q_{ \pm}$, we obtain $Q=\mathrm{O}\left(\varepsilon h^{-5}\right)$, and thus $(2.18)$ follows from (4.1)-(4.2).

To end, we simply estimate the Fourier coefficients $E_{k}^{\mathrm{u}}$ of $E^{\mathrm{u}}=\sum_{k} E_{k}^{\mathrm{u}} \mathrm{e}^{2 \pi i k t / h}$, for $k \neq 0$, shifting along complex lines $\Im t= \pm r_{h}$, with $r_{h}=\pi / 2-h$ :

$$
E_{k}^{\mathrm{u}}=h^{-1} \mathrm{e}^{-2 \pi|k| r_{h} / h} \int_{0}^{h} E^{\mathrm{u}}\left(s \pm \mathrm{i} r_{h}\right) \mathrm{e}^{2 \pi i k s / h} \mathrm{~d} s=\varepsilon M_{k}(h)+\mathrm{O}\left(\varepsilon h^{3}, \varepsilon^{2} h^{-5}\right) \mathrm{e}^{-\pi^{2}|k| / h},
$$


where $M_{k}(h)$ stand for the Fourier coefficients of the Melnikov function, and estimate (2.19) follows readily.

(b) In [DS97, Proposition 2.6], an analogous result is proved. Therefore, we only sketch the proof. The function $S^{\mathrm{u}}(t)-t$ is analytic and $h$-periodic on the complex strip $\mathcal{I}_{h}$ introduced in (2.17). For $T / 2 \leq \Re t \leq T$, using the Flow Box Theorem together with the Extension Theorem we get

$S^{\mathrm{u}}(t)-t=S\left(z^{\mathrm{u}}(t)\right)-t=S^{0}\left(z^{\mathrm{u}}(t)\right)-t+\mathrm{O}(\varepsilon)=S^{0}\left(z^{0}(t)\right)-t+\mathrm{O}\left(\varepsilon h^{-3}, \varepsilon\right)=\mathrm{O}\left(\varepsilon h^{-3}\right)$,

since $S^{0}\left(z^{0}(t)\right)=t$, as an special case of (2.12). On the one hand, estimating now the Fourier coefficients of $S^{\mathrm{u}}(t)-t$ and also those of its derivative (which has zero mean), we arrive at

$$
S^{\mathrm{u}}(t)-t=S_{0}^{\mathrm{u}}+\mathrm{O}\left(\varepsilon h^{-3}\right) \mathrm{e}^{-\pi^{2} / h}, \quad \mathrm{~d} S^{\mathrm{u}}(t) / \mathrm{d} t-1=\mathrm{O}\left(\varepsilon h^{-4}\right) \mathrm{e}^{-\pi^{2} / h}, \quad \forall t \in \mathbb{R} .
$$

Therefore $S^{\mathrm{u}}(t)$ can be inverted for real $t$. On the other hand, repeating the computations above for real $t$, but using estimate (2.15), instead of the worst one (2.14), we get

$$
S^{\mathrm{u}}(t)-t=\mathrm{O}(\varepsilon), \quad t \in \mathbb{R}
$$

and in particular $S_{0}^{\mathrm{u}}=\mathrm{O}(\varepsilon)$ for the zero order Fourier coefficient $S_{0}^{\mathrm{u}}$ of $S^{\mathrm{u}}(s)-s$ that appears in formula (4.4).

Finally, using formulae (4.4) and (4.5), we obtain for the inverse function $s=s^{\mathrm{u}}(t)$ of $s=S^{\mathrm{u}}(t)$ that $\phi^{\mathrm{u}}(s)=s^{\mathrm{u}}(s)-s$ is $\mathrm{O}(\varepsilon)$, analytic, and $h$-periodic.

(c) Formula (2.20) was proved in [DR96] for even polynomial perturbative potentials $V(y)$. Unfortunately, the reasoning given there does not apply to a series as in (1.2), and we must change our approach. Along this proof, we do not assume that $V_{1}=V^{\prime \prime}(0)=0$ because it is not used.

From formula (1.10), $M(t)$ is odd real and $h$-periodic, and its Fourier expansion can be written in the following way

$$
M(t)=\sum_{k \in \mathbb{Z}} M_{k}(h) \exp (2 \pi k \mathrm{i} t / h)=\sum_{k \geq 1} m_{k}(h) \sin (2 \pi k t / h),
$$

where $M_{0}(h)=0$ and $M_{ \pm k}(h)=\mp \frac{1}{2} m_{k}(h)$ i for some real $m_{k}(h)$, if $k \geq 1$. The idea of the proof is to get the asymptotic behaviour of the the first harmonic $m_{1}(h)$ and to bound the higher order ones. The first step is to get an uniform control on the Laurent coefficients $f_{n}(h)$ of $f(t)$ around its singularities.

Lemma 4.1 The function $f(t)=f(t, h)$ defined in (1.10) has isolated singularities just on the set $-h / 2+\pi \mathrm{i} / 2+\pi \mathrm{i} \mathbb{Z}$, being symmetric with respect to this set. Let

$$
\sum_{n \in \mathbb{Z}} f_{n}(h)\left(t-t_{\mathrm{p}}\right)^{2 n}
$$

be its Laurent expansion around the singularity $t_{\mathrm{p}}=-h / 2+\pi \mathrm{i} / 2$. Then, there exists a constant $b$ independent of $n$ and $h$ such that

$$
\left|(\mathrm{i} h)^{-2 n} f_{-n}(h)-V_{n}\right| \leq b h^{2}, \quad \forall n \geq 1,0<h \ll 1 .
$$


Proof. The first part is trivial. In order to get the second one it is very convenient to introduce the function

$$
v(\tau)=v(\tau, h)=V(\sigma(\tau, h)), \quad \sigma(\tau, h)= \begin{cases}\gamma / \sin (h \tau) & \text { if } h \neq 0 \\ \tau^{-1} & \text { if } h=0\end{cases}
$$

The singularities of $v(\tau)$ are located on the set $\pi h^{-1} \mathrm{i} \mathbb{Z}$, so $\tau=0$ is the unique singularity on the ball $|\tau|<2$, assuming $h$ small enough. Therefore, $v(\tau)$ can be expressed in terms of its Laurent series $\sum_{n \in \mathbb{Z}} v_{n}(h) \tau^{2 n}$ around $\tau=0$. From the definition of $v(\tau)$, it turns out that if $h=0, v(\tau)=\sum_{n \geq 1} V_{n} \tau^{-2 n}$, whereas if $h \neq 0, v(\tau)=$ $f\left(t_{\mathrm{p}}-\mathrm{i} h \tau\right)$. Thus

$$
v_{-n}(h)=\left\{\begin{array}{ll}
(\mathrm{i} h)^{-2 n} f_{-n}(h) & \text { if } h \neq 0 \\
V_{n} & \text { if } h=0
\end{array}, \quad \forall n \geq 1 .\right.
$$

(Here, $\tau$ is an inner-like variable, similar to the one used in [HM93] to study the behaviour of the separatrices of the standard map near its singularities.)

Now, these two results together lead to the bound

$$
\begin{aligned}
\left|(\mathrm{i} h)^{-2 n} f_{-n}(h)-V_{n}\right| & =\left|\frac{1}{2 \pi \mathrm{i}} \int_{|\tau|=1}(v(\tau, h)-v(\tau, 0)) \tau^{2 n-1} \mathrm{~d} \tau\right| \\
& \leq(2 \pi)^{-1} \int_{|\tau|=1}|v(\tau, h)-v(\tau, 0)| \mathrm{d} \tau,
\end{aligned}
$$

and the lemma follows since there exists a constant $b$ independent of $\tau$ and $h$ such that

$$
|v(\tau, h)-v(\tau, 0)| \leq b h^{2}, \quad \forall|\tau|=1,0<h \ll 1 .
$$

Indeed, a bound of this kind, with another constant, trivially holds for the function $\sigma(\tau, h)$-it is sufficient to use that $\sin z=z\left[1+\mathrm{O}\left(z^{2}\right)\right]$ and $\gamma=h\left[1+\mathrm{O}\left(h^{2}\right)\right]$-, and the composition with the entire function $V(y)$ only changes the constant, but not the bound.

During the computation of Melnikov functions for maps, elliptic functions appear in a natural way [GPB89, DR96, Lev97]. Thus, some notations must be introduced. For a general background on elliptic functions we refer to [AS72, WW27]. We follow the notation of the first reference.

Given the parameter $m \in[0,1], K=K(m)=\int_{0}^{\pi / 2}(1-m \sin \vartheta)^{-1 / 2} \mathrm{~d} \vartheta$ is the complete elliptic integral of the first kind, $K^{\prime}=K^{\prime}(m)=K(1-m)$ and $q=q(m)=\mathrm{e}^{-\pi K^{\prime} / K}$ is the nome. If any of the numbers $m, K, K^{\prime}, K^{\prime} / K$ or $q$ is given, all the rest are determined. From our purposes, it is convenient to determine the value of the quotient $K^{\prime} / K$ by imposing

$$
K^{\prime} / K=\pi / h,
$$

where $h$ is the characteristic exponent. From now on, we can consider the quantities $m$, $q$ and $K$ as functions of $h$. For instance,

$$
q=q(h)=\mathrm{e}^{-\pi^{2} / h} .
$$


Now, let us introduce the elliptic function $\psi(t)$ defined by

$$
\psi(t)=\psi(t, h)=\left(\frac{2 K}{h}\right)^{2} \mathrm{dn}^{2}\left(\frac{2 K t}{h} \mid m\right) .
$$

From $\operatorname{dn}^{2}(u \mid m)=1-m \operatorname{sn}^{2}(u \mid m)$ and the Fourier expansion of $\operatorname{sn}^{2}(u \mid m)$ given in [WW27, page 520], the Fourier expansion of $\psi(t)$ follows

$$
\psi(t)=\sum_{k \in \mathbb{Z}} \psi_{k}(h) \mathrm{e}^{2 \pi k \mathrm{i} t / h}, \quad \psi_{ \pm k}(h)=\left(\frac{2 \pi}{h}\right)^{2} \frac{k q^{k}}{1-q^{2 k}}, \quad \forall k \geq 1 .
$$

(The value of $\psi_{0}(h)$ is not needed.) The importance of $\psi(t)$ and its Fourier coefficients stems from the following key lemma.

Lemma 4.2 Let $m_{k}(h)$ be the coefficients of the expansion in sinus of the Melnikov function $M(t)$ as shown in (4.6). Then

$$
m_{k}(h)=(-1)^{k+1} 2 h \psi_{k}(h) \widehat{v}_{k}(h)=(-1)^{k+1} 8 \pi^{2} k h^{-1} \frac{q^{k}}{1-q^{2 k}} \widehat{v}_{k}(h),
$$

where

$$
\widehat{v}_{k}(h)=\sum_{n \geq 1} \frac{(2 \pi k)^{2 n-1}}{(2 n-1) !} v_{-n}(h)=\sum_{n \geq 1} \frac{(2 \pi k)^{2 n-1}}{(2 n-1) !}(\mathrm{i} h)^{-2 n} f_{-n}(h) .
$$

Proof. We note that the function

$$
f^{\prime}(t)=-\gamma \frac{\sinh (t+h / 2)}{\cosh ^{2}(t+h / 2)} V^{\prime}(\gamma \operatorname{sech}(t+h / 2))
$$

verifies the properties: (P1) it is analytic on $\mathbb{R}$ and only has isolated singularities on $\mathbb{C}$, (P2) it is $T$ i-periodic with $T=\pi$, and (P3) it is exponentially small when $|\Re t| \rightarrow \infty$. Under these hypotheses, in the Summation Formula given in [DR96, Proposition 3.1] it is stated that

$$
M(t)=\sum_{n \in \mathbb{Z}} f^{\prime}(t+h n)=-\sum_{z \in \mathcal{S}} \operatorname{res}\left\{\chi(z-t) f^{\prime}(z) ; z\right\}
$$

where $\mathcal{S}$ is the set of singularities of $f^{\prime}(z)$ on the complex strip $\{0<\Im z<\pi\}$, and $\chi(z)$ is a meromorphic function such that $\chi^{\prime}(z)=$ constant $+\psi(\pi \mathrm{i} / 2-z)$.

In our case, $\mathcal{S}$ consists of only one point $z_{\mathrm{p}}=t_{\mathrm{p}}=-h / 2+\pi \mathrm{i} / 2$. Thus, using that $\operatorname{res}\left\{f(z) ; z_{\mathrm{p}}\right\}=0$, and the Fourier expansion of $\psi$, we get

$$
\begin{aligned}
\sum_{k \in \mathbb{Z}} M_{k}(h) \mathrm{e}^{2 \pi k \mathrm{i} t / h} & =M(t) \\
& =-\operatorname{res}\left\{\chi(z-t) f^{\prime}(z) ; z_{\mathrm{p}}\right\} \\
& =\operatorname{res}\left\{\chi^{\prime}(z-t) f(z) ; z_{\mathrm{p}}\right\} \\
& =\operatorname{res}\left\{\psi\left(t+h / 2-\left(z-z_{\mathrm{p}}\right)\right) f(z) ; z_{\mathrm{p}}\right\} \\
& =\sum_{k \in \mathbb{Z}}(-1)^{k} \psi_{k}(h) \cdot \operatorname{res}\left\{\exp \left[-\frac{2 \pi k \mathrm{i}}{h}\left(z-z_{\mathrm{p}}\right)\right] \cdot f(z) ; z_{\mathrm{p}}\right\} \cdot \mathrm{e}^{2 \pi k \mathrm{i} t / h} .
\end{aligned}
$$


After a little of algebra, one obtains

$$
\operatorname{res}\left\{\exp \left[-\frac{2 \pi k \mathrm{i}}{h}\left(z-z_{\mathrm{p}}\right)\right] \cdot f(z) ; z_{\mathrm{p}}\right\}=h \widehat{v}_{k}(h) \mathrm{i}, \quad \forall k \neq 0,
$$

and the lemma follows, since $M_{ \pm k}(h)=\mp \frac{1}{2} m_{k}(h) \mathrm{i}$, for all $k \geq 1$.

These previous lemmas are intended to prove the following result on the coefficients $m_{k}(h)$ of the sinus expansion of the Melnikov function $M(t)$.

Lemma 4.3 Let $\widehat{V}: \mathbb{C} \rightarrow \mathbb{C}$ be the function defined in (1.11). Let $V_{\infty}=\max \left\{V_{n}\right\}$, where $V_{n}$ are the Taylor coefficients of the function $V(y)$ defined in (1.2). Let $B=$ $32 \pi^{2} V_{\infty}$ and $r_{h}=\pi / 2-h$. Then:

(i) The following asymptotic expression for the first coefficient $m_{1}(h)$ holds

$$
m_{1}(h)=8 \pi^{2} h^{-1} \mathrm{e}^{-\pi^{2} / h}\left[\widehat{V}(2 \pi)+\mathrm{O}\left(h^{2}\right)\right] .
$$

(ii) The higher order coefficients can be bounded as follows

$$
\left|m_{k}(h)\right| \leq B h^{-1} k \exp \left(-\frac{2 \pi k r_{h}}{h}\right), \quad \forall k \geq 2,0<h \ll 1 .
$$

Proof. Using equation (4.7), the bound $\sum_{n \geq 1} u^{2 n-1} /(2 n-1) !=\sinh u \leq \mathrm{e}^{u}$, for $u>0$, and the triangle inequality, we deduce that the functions $\widehat{v}_{k}(h)$ introduced in lemma 4.2 verify

$$
\left|\widehat{v}_{k}(h)-\widehat{V}(2 \pi k)\right| \leq b \mathrm{e}^{2 \pi k} h^{2}, \quad\left|\widehat{v}_{k}(h)\right| \leq 2 V_{\infty} \mathrm{e}^{2 \pi k}, \quad \forall k \geq 1,0<h \ll 1 .
$$

(i) Let $m_{1}^{*}(h)$ be the function given implicitly by $m_{1}(h)=8 \pi^{2} h^{-1} q\left[\widehat{V}(2 \pi)+h^{2} m_{1}^{*}(h)\right]$. Remember that $q=\mathrm{e}^{-\pi^{2} / h}$, so $\lim _{h \rightarrow 0^{+}}(q / h)=0$ and $q^{2}<1 / 2$ for small positive values of $h$. Direct computations yield

$$
\begin{aligned}
\left|m_{1}^{*}(h)\right| & =\left|\frac{m_{1}(h)-8 \pi^{2} h^{-1} q \widehat{V}(2 \pi)}{8 \pi^{2} h q}\right|=h^{-2}\left|\frac{\widehat{v}_{1}(h)}{1-q^{2}}-\widehat{V}(2 \pi)\right| \\
& \leq h^{-2}\left[\left|\widehat{v}_{1}(h)-\widehat{V}(2 \pi)\right|+\frac{q^{2}}{1-q^{2}}\left|\widehat{v}_{1}(h)\right|\right] \leq \mathrm{e}^{2 \pi}\left[b+4 V_{\infty}(q / h)^{2}\right]=\mathrm{O}(1) .
\end{aligned}
$$

(ii) We bound the coefficients $m_{k}(h)$ in the following way

$$
\left|m_{k}(h)\right|=8 \pi^{2} h^{-1} \frac{k q^{k}}{1-q^{2 k}}\left|\widehat{v}_{k}(h)\right| \leq 32 \pi^{2} V_{\infty} h^{-1} k q^{k} \mathrm{e}^{2 \pi k}=B h^{-1} k \exp \left(-\frac{2 \pi k r_{h}}{h}\right),
$$

for all $k \geq 2$ and $h>0$ small enough. 
Now, we are ready to finish the proof of proposition 2.2, taking into account the last lemma. We split the Melnikov function as $M(t)=M^{[1]}(t)+M^{[\geq 2]}(t)$, where

$$
M^{[1]}(t)=m_{1}(h) \sin (2 \pi t / h), \quad M^{[\geq 2]}(t)=\sum_{k \geq 2} m_{k}(h) \sin (2 \pi k t / h) .
$$

On the one hand, the term $M^{[1]}(t)$ verifies

$$
M^{[1]}(t)=8 \pi^{2} h^{-1} \mathrm{e}^{-\pi^{2} / h}\left[\widehat{V}(2 \pi)+\mathrm{O}\left(h^{2}\right)\right] \sin (2 \pi t / h) .
$$

On the other hand, we bound the term $M^{[\geq 2]}(t)$, for real values of $t$ and small values of $h$, in the following way

$$
\begin{aligned}
\left|M^{[\geq 2]}(t)\right| & \leq B h^{-1} \sum_{k \geq 2} k \exp \left(-2 \pi k r_{h} / h\right)=B h^{-1} \frac{\left(2-\exp \left(-2 \pi r_{h} / h\right)\right) \exp \left(-4 \pi r_{h} / h\right)}{\left(1-\exp \left(-2 \pi r_{h} / h\right)\right)^{2}} \\
& =\mathrm{O}\left(h^{-1} \exp \left(-2 \pi^{2} / h\right)\right) \leq \mathrm{O}\left(h \exp \left(-\pi^{2} / h\right)\right),
\end{aligned}
$$

and the formula (2.20) holds, as we wanted. Formula (2.21) can be obtained in a similar way.

\section{A Proof of the Upper Bound Theorem}

Along this paper, we have worked inside a complex strip $|\Im t| \leq \pi / 2-\delta$ with a variable $\delta \geq h$. The computations with a fixed $\delta$ are easier and give rise to upper bounds for the splitting of separatrices. Nevertheless, this appendix is devoted to show that the Upper Bound Theorem is a direct corollary of the theorem about exponentially small upper bounds of the splitting size for area preserving analytic maps close to the identity given in [FS90, Fon95]. We recall this theorem in a suitable form for our purposes.

Theorem A.1 Let $\mathcal{G}_{h}: \mathbb{R}^{2} \rightarrow \mathbb{R}$ be a family of analytic area preserving diffeomorphisms with $h>0$ having the form

$$
\mathcal{G}_{h}(\zeta)=\left(\begin{array}{cc}
\mathrm{e}^{h} & 0 \\
0 & \mathrm{e}^{-h}
\end{array}\right) \zeta+h\left[\mathcal{G}^{0}(\zeta)+h^{q} \mathcal{G}^{1}(\zeta, h)\right], \quad \mathcal{G}^{0}, \mathcal{G}^{1}=\mathrm{O}\left(\zeta^{2}\right)
$$

where $\zeta=\left(\zeta_{1}, \zeta_{2}\right)$, and $q>0$. Assume that the vector field

$$
\dot{\zeta}=\mathcal{X}^{0}(\zeta), \quad \mathcal{X}^{0}(\zeta)=\left(\zeta_{1},-\zeta_{2}\right)+\mathcal{G}^{0}(\zeta)
$$

verifies the following properties:

(i) It has a homoclinic orbit $\zeta^{0}$ to the origin analytic on $\mathcal{I}_{\delta_{0}}:=\left\{t \in \mathbb{C}:|\Im t|<\delta_{0}\right\}$.

(ii) There exists $\delta_{1} \in\left(0, \delta_{0}\right)$ such that the maps $\mathcal{G}_{h}(\zeta)$ are analytic on a neighbourhood of $\zeta^{0}\left(\mathcal{I}_{\delta_{1}}\right)$, for small enough values of $h$. 
Then, given any $\delta \in\left(0, \delta_{1}\right)$, there exists a constant $N=N(\delta)$ such that

$$
\text { splitting size } \leq N(\delta) \mathrm{e}^{-2 \pi \delta / h} \quad\left(h \rightarrow 0^{+}\right) .
$$

Remark A.1 Hypothesis (i) is a necessary and sufficient condition for the existence of a constant $h_{0}>0$ such that $\mathcal{G}_{h}$ has homoclinic points for all $h \in\left(0, h_{0}\right)$, see [Fon89]. Thus, it makes sense to speak of splitting size under the above assumptions.

Now, we are ready to sketch the proof of the Upper Bound Theorem. The notations in the statement of theorem A.1 must be kept in mind.

Setting $\varepsilon=\mathrm{O}\left(h^{p}\right)$ in equation (1.1), the linear change of variables (2.1) conjugates $F(z)$ to an analytic area preserving diffeomorphism having the form (A.1), where

$$
\mathcal{G}^{0}(\zeta)=\left(-\left(\zeta_{1}+\zeta_{2}\right)^{3},\left(\zeta_{1}+\zeta_{2}\right)^{3}\right) .
$$

Then, the vector field (A.2) verifies the hypotheses (i)-(ii) for any $0<\delta_{1}<\delta_{0}=\pi / 2$. Namely, since its homoclinic orbit $\zeta^{0}(t)$ is given by

$$
\zeta^{0}(t)=\left(\frac{\cosh t-\sinh t}{2 \cosh ^{2} t}, \frac{\cosh t+\sinh t}{2 \cosh ^{2} t}\right),
$$

it is immediate that $\delta_{0}=\pi / 2$, and an easy computation shows that we can choose an arbitrary $\delta_{1} \in\left(0, \delta_{0}\right)=(0, \pi / 2)$, taking $h$ small enough.

Consequently, the Upper Bound Theorem follows simply noting that $\delta \in(0, \pi / 2)$ implies $\alpha=2 \pi \delta \in\left(0, \pi^{2}\right)$. The factor $\mathrm{O}\left(h^{3}\right)$ due to the linear change has no importance in front of the exponential decay of the splitting size.

To end, we want to mention that for fixed $\varepsilon$, the Upper Bound Theorem holds if and only if $\varepsilon<1 / 2 V_{2}$, where $V_{2}$ stands for the coefficient in the quartic term of the Taylor expansion of the perturbative potential $V(y)$. The point is that for fixed $\varepsilon$, one has

$$
\mathcal{G}^{0}(\zeta)=a\left(-\left(\zeta_{1}+\zeta_{2}\right)^{3},\left(\zeta_{1}+\zeta_{2}\right)^{3}\right), \quad a=1-2 V_{2} \varepsilon,
$$

so that the vector field (A.2) has a homoclinic orbit to the origin if and only if $a>0$. We omit the details.

\section{Acknowledgements}

This work has been partially supported by the EC grant ERBCHRXCT-940460. Research by A. Delshams is also supported by the Spanish grant DGICYT PB94-0215 and the Catalan grant CIRIT 1996SGR-00105. Research by R. Ramírez-Ros is also supported by the U.P.C. grant PR9409. The authors are indebted to R. de la Llave and C. Simó for very useful remarks and comments. 


\section{References}

[ACKR92] C. Amick, S.C.E. Ching, L.P. Kadanoff, and V. Rom-Kedar. Beyond all orders: Singular perturbations in a mapping. J. Nonlinear Sci., 2:9-67, 1992.

[Ang93] S. Angenent. A variational interpretation of Melnikov's function and exponentially small separatrix splitting. In D. Salamon, editor, Symplectic geometry, volume 192 of London Math. Soc. Lecture Note Ser., pages 5-35. Cambridge Univ. Press, 1993.

[AS72] M. Abramowitz and I. Stegun. Handbook of Mathematical Functions. Dover, 1972.

[Che95] V.L. Chernov. Resurgence of separatrices of the quadratic map. Math. Preprints Series 185, Univ. Barcelona, November 1995.

[Cus78] R. Cushman. Examples of nonintegrable analytic Hamiltonian vectorfields with no small divisors. Trans. Amer. Math. Soc., 238(1):45-55, 1978.

[De195] D. Delatte. On normal forms in Hamiltonian dynamics, a new approach to convergence questions. Ergodic Theory Dynam. Systems, 15:49-66, 1995.

[DGJS97] A. Delshams, V.G. Gelfreich, A. Jorba, and T.M. Seara. Exponentially small splitting of separatrices under fast quasiperiodic forcing. Comm. Math. Phys., 189(1):35$71,1997$.

[DMS97] A. Delshams, A. Mir, and T.M. Seara. Psi-series and splitting of separatrices. Preprint in progress, 1997.

[DR96] A. Delshams and R. Ramírez-Ros. Poincaré-Melnikov-Arnold method for analytic planar maps. Nonlinearity, 9(1):1-26, 1996.

[DR97a] A. Delshams and R. Ramírez-Ros. Melnikov potential for exact symplectic maps. To appear in Comm. Math. Phys., 1997.

[DR97b] A. Delshams and R. Ramírez-Ros. Singular separatrix splitting and Melnikov method: An experimental approach. Preprint, 1997.

[DS92] A. Delshams and T.M. Seara. An asymptotic expression for the splitting of separatrices of the rapidly forced pendulum. Comm. Math. Phys., 150:433-463, 1992.

[DS97] A. Delshams and T.M. Seara. Splitting of separatrices in Hamiltonian systems with one and a half degrees of freedom. Math. Phys. Electron. J., 3(4):1-40, 1997.

[Fon89] E. Fontich. On the existence of homoclinic points for conservative diffeomorphisms. In C. Alsina, J. Llibre, Ch. Mira, C. Simó, G. Targonski, and R. Thibault, editors, European Conference on Iteration Theory (ECIT 87), pages 189-197. Held in Caldes de Malavella, Spain, 20-26 September, 1987. World Scientific, Singapore, 1989.

[Fon93] E. Fontich. Exponentially small upper bounds for the splitting of separatrices for high frequency periodic perturbations. Nonlinear Anal., 20(6):733-744, 1993.

[Fon95] E. Fontich. Rapidly forced planar vector fields and splitting of separatrices. $J$. Differential Equations, 119(2):310-335, 1995. 
[FS90] E. Fontich and C. Simó. The splitting of separatrices for analytic diffeomorphisms. Ergodic Theory Dynam. Systems, 10:295-318, 1990.

[FS96] B. Fiedler and J. Scheurle. Discretization of homoclinic orbits, rapid forcing and "invisible" chaos. Mem. Amer. Math. Soc., 119(570), 1996. viii + 79 pp.

[Ga194] G. Gallavotti. Twistless KAM tori, quasi flat homoclinic intersections, and other cancellations in the perturbation series of certain completely integrable Hamiltonian systems. A review. Rev. Math. Phys., 6(3):343-411, 1994.

[Gel93] V.G. Gelfreich. Separatrices splitting for the rapidly forced pendulum. In S. Kuksin, V.F. Lazutkin, and J. Pöschel, editors, Proceedings of the Dynamical Systems Semester, pages 47-67. Held in St. Petersburg, Russia, 17-30 November, 1991. Birkhäuser, Basel-Boston-Stuttgart, 1993.

[Gel96] V.G. Gelfreich. Conjugation to a shift and the splitting of invariant manifolds. Appl. Math. (Warsaw), 24(2):127-140, 1996.

[Gel97] V.G. Gelfreich. Melnikov method and exponentially small splitting of separatrices. Phys. D, 101(3-4):227-248, 1997.

[GLS94] V.G. Gelfreich, V.F. Lazutkin, and N.V. Svanidze. A refined formula for the separatrix splitting for the standard map. Phys. D, 71:82-101, 1994.

[GLT91] V.G. Gelfreich, V.F. Lazutkin, and M.B. Tabanov. Exponentially small splitting in Hamiltonian systems. Chaos, 1(2):137-142, 1991.

[GPB89] M.L. Glasser, V.G. Papageorgiou, and T.C. Bountis. Melnikov's function for twodimensional mappings. SIAM J. Appl. Math., 49(3):692-703, 1989.

[HM93] V. Hakim and K. Mallick. Exponentially small splitting of separatrices, matching in the complex plane and Borel summation. Nonlinearity, 6:57-70, 1993.

[HMS88] P. Holmes, J. Marsden, and J. Scheurle. Exponentially small splittings of separatrices with applications to KAM theory and degenerate bifurcations. Contemp. Math., 81:213-244, 1988.

[Las78] L.J. Laslett. Some illustrations of stochasticity. In S. Jorna, editor, Topics in Nonlinear Dynamics, a Tribute to Sir Edward Bullard, number 46 in AIP Conference Proceedings, pages 221-243. Amer. Inst. Phys., New York, 1978.

[Laz84] V.F. Lazutkin. Splitting of separatrices for the Chirikov's standard map. Preprint 6372-84, VINITI, 1984. (In Russian).

[Laz91] V.F. Lazutkin. On the width of the instability zone near the separatrices of a standard mapping. Soviet Math. Dokl., 42(1):5-9, 1991.

[Laz93] V.F. Lazutkin. Resurgent approach to the separatrices splitting. In C. Perelló, C. Simó, and J. Solà-Morales, editors, International Conference on Differential Equations (Equa $\frac{\partial i}{\partial t} f f$ 91), pages 163-176. Held in Barcelona, 26-31 August, 1991. World Scientific, Singapore, 1993. 
[Lev97] P. Levallois. Calcul d'une fonction de Melnikov et de ses zeros pour une perturbation algébrique du billiard elliptique. Ergodic Theory Dynam. Systems, 17:435-444, 1997.

[LM96] H.E. Lomelí and J.D. Meiss. Heteroclinic orbits, and transport in a perturbed integrable standard map. Preprint 96-77, mp_arc@math .utexas . edu, February 1996.

[Lom96] H.E. Lomelí. Perturbations of elliptic billiards. Phys. D, 99:59-80, 1996.

[LST89] V.F. Lazutkin, I.G. Schachmannski, and M.B. Tabanov. Splitting of separatrices for standard and semistandard mappings. Phys. D, 40:235-248, 1989.

[LT93] P. Levallois and M.B. Tabanov. Séparation des séparatrices du billard elliptique pour une perturbation algébrique et symétrique de l'ellipse. C. R. Acad. Sci. Paris Sér. I Math., 316:589-592, 1993.

[McM71] E.M. McMillan. A problem in the stability of periodic systems. In E. Brittin and H. Odabasi, editors, Topics in modern physics, a tribute to E.V. Condon, pages 219-244. Colorado Assoc. Univ. Press, Boulder, CO, 1971.

[Mei92] J.D. Meiss. Symplectic maps, variational principles, and transport. Rev. Modern Phys., 64(3):795-848, 1992.

[Mos56] J. Moser. The analytic invariants of an area-preserving mapping near a hyperbolic fixed point. Comm. Pure Appl. Math., 9:673-692, 1956.

[Nei84] A.I. Neishtadt. The separation of motions in systems with rapidly rotating phase. J. Appl. Math. Mech., 48(2):133-139, 1984.

[Poi99] H. Poincaré. Les méthodes nouvelles de la mécanique céleste, volume 1, 2, 3. Gauthier-Villars, Paris, 1892-1899.

[RW88] V. Rom-Kedar and S. Wiggins. Transport in two-dimensional maps. Arch. Rational Mech. Anal., 109:239-298, 1988.

[RW97] M. Rudnev and S. Wiggins. Existence of exponentially small separatrix splittings and homoclinic connections between whiskered tori in weakly hyperbolic near-integrable Hamiltonian systems. To appear in Phys. D.

[Sau95] D. Sauzin. Résurgence paramétrique et exponentielle petitese de l'ecart des séparatrices du pendule rapidament forcé. Ann. Inst. Fourier (Grenoble), 45(2):453$511,1995$.

[Sim94] C. Simó. Averaging under fast quasiperiodic forcing. In J. Seimenis, editor, Hamiltonian Mechanics: Integrability and Chaotic Behaviour, volume 331 of NATO Adv. Sci. Inst. Ser. B Phys., pages 13-34. Held in Torun, Polland, 28 June-2 July 1993. Plenum, New York, 1994.

[SS96] B.Y. Sternin and V.E. Shatalov. Borel-Laplace transform and asymptotic theory: Introduction to resurgent analysis. CRC Press, Boca Raton, Florida, 1996.

[Sur89] Y.B. Suris. Integrable mappings of the standard type. Functional Anal. Appl., 23:74-76, 1989 . 
[Sur94] Y.B. Suris. On the complex separatrices of some standard-like maps. Nonlinearity, 7:1225-1236, 1994.

[Tab94] M.B. Tabanov. Separatrices splitting for Birkhoff's billiard in a symmetric convex domain, close to an ellipse. Chaos, 4(4):595-606, 1994.

[Tre96a] D.V. Treschev. An averaging method for Hamiltonian systems, exponentially close to integrable ones. Chaos, 6(1):6-14, 1996.

[Tre96b] D.V. Treschev. Width of stochastic layers in near-integrable two-dimensional symplectic maps. Preprint, 1996.

[Tre97] D.V. Treschev. Separatrix splitting for a pendulum with rapidly oscillating suspension point. Russ. J. Math. Phys, 5(1):63-98, 1997.

[WW27] E.T. Whittaker and G.N. Watson. A course of modern analysis. Cambridge Univ. Press, Cambridge, 1927.

\section{Internet access:}

All the authors' quoted preprints are available at http://www-ma1.upc.es in the Preprints pages, or at ftp://ftp-ma1.upc.es, in the pub/preprints directory.

E-mail addresses of the authors: amadeu@ma1.upc.es, rafael@tere.upc.es. 